\title{
The Approach of Moments for Polynomial Equations
}

\author{
Monique Laurent ${ }^{1}$ and Philipp Rostalski ${ }^{2}$ \\ 1 Centrum voor Wiskunde en Informatica, Science Park 123, 1098 XG \\ Amsterdam, and Tilburg University, P.O. Box 90153, 5000 LE Tilburg, \\ Netherlands. M.Laurent@cwi.nl \\ 2 Department of Mathematics, UC Berkeley, 1067 Evans Hall, Berkeley, CA \\ 94720-3840, USA. philipp@math. berkeley.edu
}

Summary. In this chapter we present the moment based approach for computing all real solutions of a given system of polynomial equations. This approach builds upon a lifting method for constructing semidefinite relaxations of several nonconvex optimization problems, using sums of squares of polynomials and the dual theory of moments. A crucial ingredient is a semidefinite characterization of the real radical ideal, consisting of all polynomials with the same real zero set as the system of polynomials to be solved. Combining this characterization with ideas from commutative algebra, (numerical) linear algebra and semidefinite optimization yields a new class of real algebraic algorithms. This chapter sheds some light on the underlying theory and the link to polynomial optimization.

\section{Introduction}

Computing all points $x \in \mathbb{K}^{n}(\mathbb{K}=\mathbb{R}$ or $\mathbb{C}$ ) at which a given system of polynomials in $n$ variables

$$
h_{1}, \ldots, h_{m} \in \mathbb{R}\left[x_{1}, \ldots, x_{n}\right]=\mathbb{R}[x]
$$

vanishes simultaneously, is an old problem arising in many mathematical models in science and engineering, with numerous applications in different areas ranging from control, cryptography, computational geometry, coding theory and computational biology to optimization, robotics, statistics and many others (see, e.g., [44]). In this chapter we will focus on the characterization and the (numerical) computation of all real roots or, more generally, of all roots lying in some given basic semi-algebraic set, i.e. satisfying some prescribed polynomial inequalities. A variety of methods has been proposed to tackle such problems, some of which will be briefly recalled in the next section. In this chapter we will focus on a new approach based on sums of squares of polynomials and the dual theory of moments. In this context, semidefinite 
programming will be the tool permitting to distinguish algorithmically between real and complex nonreal elements.

\subsection{Existing methods}

Solving polynomial equations has a long tradition covered in a vast literature; for information and further references see e.g. the monographs of Basu, Pollack and Roy [2], Dickenstein and Emiris [9], Mora [27, 28], Elkadi and Mourrain [10], Stetter [43], Sturmfels [44]. We do not attempt a complete description of all existing methods, but instead we only try to give a coarse classification. Most existing algorithms can be roughly categorized according to the following criteria: local vs. global search, numerical vs. exact/symbolic computation, and solving over the complex numbers vs. solving over the real numbers.

\section{Over the complex numbers}

Symbolic methods. Gröbner bases, resultants or, more generally, border bases and generalized normal form algorithms are typical representatives of this class of methods. The main idea is to compute the structure of the quotient algebra $\mathbb{R}[x] / I$ (where $I$ is the ideal generated by the given polynomials $h_{i}$ ) and to use this information to characterize the roots, e.g., using the shape lemma, or Stickelberger's theorem (viz. the eigenvalue method), or the rational univariate representation.

The following basic fact plays a crucial role: The system of polynomial equations $h_{1}=\cdots=h_{m}=0$ has finitely many roots if and only if the quotient ring $\mathbb{R}[x] / I$ of the underlying ideal $I=\left\langle h_{1}, \ldots, h_{m}\right\rangle$ is finite dimen-

sional as a vector space. This in turn enables to reduce the computation of all complex roots to tasks of finite dimensional linear algebra (like eigenvalue computations). Roughly speaking, the basic idea is to replace the given system $h_{i}=0$ by a new equivalent system $g_{j}=0$ with the same set of complex roots, but with a much easier structure facilitating the extraction of the roots.

For instance, one may find an equivalent system comprising polynomials in triangular form $g_{1} \in \mathbb{R}\left[x_{1}\right], g_{2} \in \mathbb{R}\left[x_{1}, x_{2}\right], \ldots, g_{n} \in \mathbb{R}\left[x_{1}, \ldots, x_{n}\right]$, which can be solved by solving a sequence of univariate root finding problems. Such an approach suffers however from the propagation of numerical errors and triangular representations are difficult to compute, typically involving lexicographic Gröbner bases. A more efficient approach is the rational univariate representation, where the new system has a parametric representation:

$$
x_{1}=h_{1}(t) / h(t), \ldots, x_{n}=h_{n}(t) / h(t), f(t)=0 \quad\left(h_{i}, h, f \in \mathbb{R}[t]\right),
$$

which requires the solution of a single univariate polynomial: $f(t)=0$ (see $[38])$.

Symbolic-numeric methods. Motivated by the great success of numerical linear algebra, a new trend in applied mathematics is to carefully combine 
symbolic methods (mostly border bases methods) with numerical calculations, such as singular value decomposition, LU-factorization and other workhorses of numerical linear algebra in order to derive powerful algorithms for large scale problems (see e.g. [30] for details). As mentioned above, symbolic methods are able to transform the given system $h_{i}=0$ into a new, better structured system $g_{j}=0$. Then the task of computing the complex roots is reduced to (numerical) linear algebra, like computing the eigenvalues/eigenvectors of companion matrices (cf. Section 2.2 below), or univariate root finding.

Numerical methods. The most successful approach in this class of methods is homotopy continuation. Such methods rely on Bertini's theorem allowing to deform an easier instance with known solutions of the class of problems to be solved into the original system, without encountering singularities along the path (cf. [40] for details). Keeping track of the roots during this deformation allows to compute the desired roots.

\section{Over the real numbers}

While the task of solving polynomial equations over the complex numbers is relatively well understood, computing only the real roots is still largely open. The need for methods tailored to real root finding is mainly motivated by applications, where often only the real roots are meaningful, and whose number is typically much smaller than the total number of complex solutions. As an illustration, just consider the simple equation $x_{1}^{2}+x_{2}^{2}=0$, where not even the dimensions of the real and complex solution sets agree!

So far, real solving methods were mostly build upon local methods combined with a bisection search strategy. More recently, two new global approaches have been considered which can be seen as refinements of complex root finding methods mentioned above: the SDP based moment approach (which is the focus of this chapter), and a new homotopy continuation method tuned to real roots. The three main classes of methods for real roots are:

Subdivision methods. Combining exclusion criteria to remove parts of the search space not containing any real root and identify regions containing isolated real roots, with local search strategies such as Newton-Raphson or higher order methods are the basis for the class of subdivision methods. The search space is subdivided until it contains only a single root and Newton's method converges (cf. e.g. [31] for a recent account). Exclusion criteria include real root counting techniques based e.g. on Sturm-Habicht sequences, Descartes' rule of signs (for univariate polynomials), or signatures of Hermite forms (in the multivariate case). Such techniques, combined with deformation techniques using Puiseux series, are also extended to the problem of computing at least one point in each connected component of an algebraic variety (possibly of positive dimension) (cf. [2] for a detailed account).

Khovanskii-Rolle continuation. This method is a recent extension of curve following methods (like homotopy continuation for complex roots) tailored to 
real roots. It exploits the fact that there are sharp bounds for the number of real roots of systems of equations with few monomials, combined with Gale duality. The approach allows to track significantly fewer paths of an auxiliary system leading to all nondegenerate real solutions of the original system. It is still under investigation, but has the potential to become an efficient algorithm for real root finding (see $[3,41]$ for details).

Moment methods. This class of methods was first proposed in [17] with extensions in $[18,19]$, and is the focus of this chapter. The basic idea is to compute the real roots by working in a smaller quotient space, obtained by taking the quotient by the real radical ideal $\sqrt[\mathbb{R}]{I}$ of the original ideal $I$, consisting of all polynomials that vanish at the set of common real roots of the original system $h_{i}=0$. In this way, computing the real roots is again reduced to a task of numerical linear algebra, now in the finite dimensional vector space $\mathbb{R}[x] / \sqrt[\mathbb{R}]{I}$ (assuming only that the number of real roots is finite, while the total number of complex roots could be infinite). Finding the real radical ideal is achieved by computing the kernel of a generic moment matrix obtained by solving iteratively certain semidefinite programming problems.

\subsection{The basic idea of the moment method}

Most symbolic and symbolic/numeric algorithms for solving a system of polynomials decompose the structure of the polynomial ring into its ideal structure (namely, the ideal $I$ generated by the equations to be solved) and its vector space structure (corresponding to the quotient of the polynomial ring by this ideal). While the former is treated with symbolic methods one can use efficient linear algebra for the latter. We start with an elementary introduction. Let

$$
h_{1}(x)=\cdots=h_{m}(x)=0
$$

be the system of polynomial equations to be solved. Denote by $D \in \mathbb{N}$ the maximum degree of the polynomials $h_{i}$ and let $I=\left\langle h_{1}, \ldots, h_{m}\right\rangle$ be the ideal generated by these polynomials, i.e., the set of all polynomials $\sum_{i} u_{i} h_{i}$ with $u_{i} \in \mathbb{R}[x]$. If we form the matrix $H$ whose rows are the coefficient vectors of the polynomials $h_{i}$, then the roots of the system (1) are precisely the elements $x \in \mathbb{C}^{n}$ satisfying $H[x]_{D}=0$, where for any integer $t \in \mathbb{N}$,

$$
[x]_{t}=\left(1, x_{1}, \ldots, x_{n}, x_{1}^{2}, x_{1} x_{2}, \ldots, x_{n}^{t}\right)
$$

denotes the vector of all monomials of degree at most $t$. Augmenting the system (1) with new polynomials obtained by multiplying the $h_{i}$ 's by monomials does not change its set of common roots. Given an integer $t$, we add all possible multiples of the $h_{i}$ 's with degree at most $t$, i.e., we add all 'valid' equations: $x^{\alpha} h_{i}=0$ where $|\alpha| \leq t-\operatorname{deg}\left(h_{i}\right)$. This yields a new, larger system of polynomials whose coefficient vectors make the rows of a matrix $\tilde{H}_{t}$ (known as Sylvester or Macaulay-like matrix). Again, the roots of (1) are those elements $x \in \mathbb{C}^{n}$ satisfying $\tilde{H}_{t}[x]_{t}=0$. 
The basic idea is to linearize this system of equations by introducing variables $y=\left(y_{\alpha}\right)$ for the monomials $x^{\alpha}$ and to solve instead a linear system:

$$
\tilde{H}_{t} y=0 \text {. }
$$

The kernel of the matrix $\tilde{H}_{t}$ is a linear subspace, which contains the vectors $[x]_{t}$ for all roots $x$ of the system (1) and thus also their linear span. When the system (1) has finitely many complex roots, it turns out that, for $t$ large enough, (some projection of) the kernel of $\tilde{H}_{t}$ coincides with the linear span of the monomial vectors corresponding to the roots of (1), which opens the way to extracting the roots. More precisely, the central observation (dating back to [23]) is that for $t$ large enough a Gaussian elimination on the Sylvester matrix $\tilde{H}_{t}$ will reveal a Gröbner basis for the ideal $I$ and thus the desired quotient ring structure $\mathbb{R}[x] / I$. This in turn can be used to reduce the multivariate root finding problem to a simple eigenvalue calculation (as recalled in Section 2.2).

If we want to compute the real roots only, we need a mechanism to cancel out all (or as many as possible) nonreal solutions among the complex ones. This cancellation can be done by augmenting the original system (1) with additional polynomials derived from sums of squares of polynomials in the ideal $I$. We introduce this idea by means of a simple example.

Example 1. Consider the ideal $I \subseteq \mathbb{R}\left[x_{1}, x_{2}\right]$ generated by the polynomial $h=x_{1}^{2}+x_{2}^{2}$. The complex variety is positive dimensional, since it consists of infinitely many complex roots: $x_{2}= \pm \mathbf{i} x_{1}\left(x_{1} \in \mathbb{C}\right)$, while the origin $(0,0)$ is the only real root. If we add the two polynomials $p_{1}=x_{1}, p_{2}=x_{2}$ to $I$ the real variety remains unchanged, but none of the complex nonreal roots survives this intersection. Note that $p_{1}, p_{2}$ have the property that the polynomial $p_{1}^{2}+p_{2}^{2}=h$ is a sum of squares of polynomials belonging to $I$.

This example illustrates the following fact: If the $p_{i}$ 's are polynomials for which $\sum_{i} p_{i}^{2} \in I$, then each $p_{i}$ vanishes at all the real roots of the ideal $I$ (but not necessarily at its complex nonreal roots!). Thus we can add the $p_{i}$ 's to the original system (1) without altering its set of real roots. The formal tool behind this augmentation is the Real Nullstellensatz (see Theorem 1), which states that the set of real solutions to the system (1) remains unchanged if we add to it any polynomial appearing with an even degree in a sum of squares polynomial that belongs to $I$. The set of all such polynomials is known as the real radical ideal of $I$, denoted as $\sqrt[\mathbb{R}]{I}$ (see Section 2 for definitions). A main feature of the moment matrix method is that it permits to generate the polynomials in the real radical ideal in a systematic way, using duality.

Let us first look directly at the additional properties that are satisfied by a vector $y=[x]_{t} \in \operatorname{Ker} \tilde{H}_{t}$, when $x$ is a real root of (1). Obviously the matrix $[x]_{s}[x]_{s}^{T}$ is positive semidefinite for any integer $s$ and by 'linearizing' (replacing $x^{\alpha}$ by $y_{\alpha}$ ) we obtain the following matrix of generalized Hankel type: $M_{s}(y)=\left(y_{\alpha+\beta}\right)_{\alpha, \beta \in \mathbb{N}_{s}^{n}}$. Matrices with this generalized Hankel structure 
are also known as moment matrices (see Definition 3). As an illustration we display $M_{s}(y)$ for the case $n=2$ :

$$
[x]_{s}[x]_{s}^{T}=\left(\begin{array}{ccccc}
1 & x_{1} & x_{2} & x_{1}^{2} & \ldots \\
x_{1} & x_{1}^{2} & x_{1} x_{2} & x_{1}^{3} & \ldots \\
x_{2} & x_{1} x_{2} & x_{2}^{2} & x_{1}^{2} x_{2} & \ldots \\
x_{1}^{2} & x_{1}^{3} & x_{1}^{2} x_{2} & x_{1}^{4} & \ldots \\
\vdots & \vdots & \vdots & \vdots & \ddots
\end{array}\right) \rightsquigarrow M_{s}(y)\left(\begin{array}{ccccc}
1 & y_{10} & y_{01} & y_{20} & \ldots \\
y_{10} & y_{20} & y_{11} & y_{30} & \ldots \\
y_{01} & y_{11} & y_{02} & y_{21} & \ldots \\
y_{20} & y_{30} & y_{21} & y_{40} & \ldots \\
\vdots & \vdots & \vdots & \vdots & \ddots
\end{array}\right) .
$$

Therefore, we can restrict the search in the kernel of the Sylvester matrix $\tilde{H}_{t}$ to the vectors $y$ satisfying the additional positive semidefiniteness condition: $M_{s}(y) \succeq 0$ for all $s \leq t / 2$. This condition captures precisely the 'real algebraic' nature of real numbers vs. complex numbers, as it would not be valid for vectors $y$ corresponding to complex nonreal roots.

Example 2. (Example 1 cont.) Say we wish to compute the real roots of the polynomial $h=x_{1}^{2}+x_{2}^{2}$. After linearization, the constraint $H y=0$ reads: $y_{20}+y_{02}=0$. Positive semidefiniteness requires $y_{20} \geq 0, \quad y_{02} \geq 0$ which, combined with $y_{20}+y_{02}=0$ implies $y_{20}=y_{02}=0$ and thus $y_{10}=y_{01}=$ $y_{11}=0$ (using again $M_{1}(y) \succeq 0$ ). Therefore, we find $y=(1,0,0,0,0,0)$ as the unique solution, so that $y=[x]_{2}$ corresponds to the unique real root $x=(0,0)$ of $h$. The kernel of $M_{1}(y)$ contains the vectors $(0,1,0)$ and $(0,0,1)$, which can be seen as the coefficient vectors of the two polynomials $p_{1}=x_{1}$ and $p_{2}=x_{2}$ in the monomial basis $\left\{1, x_{1}, x_{2}\right\}$ of $\mathbb{R}[x]_{1}$. In other words the kernel of $M_{1}(y)$ already contains a basis of the real radical ideal $\sqrt[\mathbb{R}]{I}$.

Although the above example is extremely simplistic, it conveys the main idea: The kernel of $M_{s}(y)$ characterizes (for $s$ large enough) the real radical ideal and plays the role of the range space of $H$ in standard normal form algorithms.

\subsection{Organization of the chapter}

First we recall some basic material from polynomial algebra in Section 2. This material can be found in most standard textbooks and is used throughout the chapter. The relation between moment matrices and real radical ideals as well as the moment method for real root finding is discussed in Section 3. This section and in particular the semidefinite characterization of the real radical ideal form the heart of the chapter. We also discuss the link to some complex root finding methods and in Section 4 we briefly touch some related topics: polynomial optimization and the study of semi-algebraic sets, emptyness certificates, positive dimensional varieties, and quotient ideals. Throughout the chapter we illustrate the results with various examples. 


\section{Preliminaries of polynomial algebra}

\subsection{Polynomial ideals and varieties}

The polynomial ring and its dual. For the sake of simplicity we deal with polynomials with real coefficients only although some results remain valid for polynomials with complex coefficients. Throughout $\mathbb{R}[x]:=\mathbb{R}\left[x_{1}, \ldots, x_{n}\right]$ denotes the ring of multivariate polynomials in $n$ variables. For $\alpha \in \mathbb{N}^{n}, x^{\alpha}$ denotes the monomial $x_{1}^{\alpha_{1}} \cdots x_{n}^{\alpha_{n}}$, with degree $|\alpha|:=\sum_{i=1}^{n} \alpha_{i}$. Set $\mathbb{N}_{t}^{n}:=$ $\left\{\alpha \in \mathbb{N}^{n}|| \alpha \mid \leq t\right\}$ and let

$$
[x]_{\infty}=\left(x^{\alpha}\right)_{\alpha \in \mathbb{N}^{n}}, \quad[x]_{t}=\left(x^{\alpha}\right)_{\alpha \in \mathbb{N}_{t}^{n}}
$$

denote the vectors comprising all monomials (resp., all monomials of degree at most $t$ ) in $n$ variables. A polynomial $p \in \mathbb{R}[x]$ can be written as $p=\sum_{\alpha \in \mathbb{N}^{n}} p_{\alpha} x^{\alpha}$ with finitely many nonzero $p_{\alpha}$ 's; its support is the set of monomials appearing with a nonzero coefficient, its (total) degree $\operatorname{deg}(p)$ is the largest degree of a monomial in the support of $p$, and $\operatorname{vec}(p)=\left(p_{\alpha}\right)$ denotes the vector of coefficients of $p$. The set $\mathbb{R}[x]_{t}$ consists of all polynomials with degree at most $t$.

Given a vector space $A$ on $\mathbb{R}$, its dual space $A^{*}$ consists of all linear functionals from $A$ to $\mathbb{R}$. The orthogonal complement of a subset $B \subseteq A$ is

$$
B^{\perp}:=\left\{L \in A^{*} \mid L(b)=0 \forall b \in B\right\}
$$

and $\operatorname{Span}_{\mathbb{R}}(B)$ denotes the linear span of $B$. Then, $\operatorname{Span}_{\mathbb{R}}(B) \subseteq\left(B^{\perp}\right)^{\perp}$, with equality when $A$ is finite dimensional. We consider here the case $A=\mathbb{R}[x]$ and $A=\mathbb{R}[x]_{t}$. Examples of linear functionals on $\mathbb{R}[x]$ are the evaluation

$$
\Lambda_{v}: p \in \mathbb{R}[x] \mapsto \Lambda_{v}(p)=p(v)
$$

at a point $v \in \mathbb{R}^{n}$ and, more generally, the differential functional

$$
\partial_{v}^{\alpha}: p \in \mathbb{R}[x] \mapsto \partial_{v}^{\alpha}(p)=\frac{1}{\prod_{i=1}^{n} \alpha_{i} !}\left(\frac{\partial^{|\alpha|}}{\partial x_{1}^{\alpha_{1}} \ldots \partial x_{n}^{\alpha_{n}}} p\right)(v),
$$

which evaluates at $v \in \mathbb{R}^{n}$ the (scaled) $\alpha$-th derivative of $p$ (where $\alpha \in \mathbb{N}$ ). For $\alpha=0, \partial_{v}^{\alpha}$ coincides with the evaluation at $v$, i.e., $\partial_{v}^{0}=\Lambda_{v}$. For $\alpha, \beta \in \mathbb{N}^{n}$,

$$
\partial_{0}^{\alpha}\left(x^{\beta}\right)=1 \text { if } \alpha=\beta \text {, and } 0 \text { otherwise. }
$$

Therefore, any linear form $\Lambda \in \mathbb{R}[x]^{*}$ can be written in the form:

$$
\Lambda=\sum_{\alpha \in \mathbb{N}^{n}} \Lambda\left(x^{\alpha}\right) \partial_{0}^{\alpha} .
$$

This is in fact a formal power series as in general infinitely many $\Lambda\left(x^{\alpha}\right)$ are nonzero. Let $y=\left(y_{\alpha}\right)$ denote the coefficient series of $\Lambda$ in $\left(\partial_{0}^{\alpha}\right)$ i.e. $y_{\alpha}=\Lambda\left(x^{\alpha}\right)$, 
such that $\Lambda(p)=y^{T} \operatorname{vec}(p)$ for all $p \in \mathbb{R}[x]$. For instance, the evaluation at $v \in \mathbb{R}^{n}$ reads $\Lambda_{v}=\sum_{\alpha} v^{\alpha} \partial_{0}^{\alpha}$, with coefficient series $[v]_{\infty}=\left(v^{\alpha}\right)_{\alpha \in \mathbb{N}^{n}}$ in $\left(\partial_{0}^{\alpha}\right)$.

Ideals and varieties. A linear subspace $I \subseteq \mathbb{R}[x]$ is an ideal if $p \in I, q \in \mathbb{R}[x]$ implies $p q \in I$. The ideal generated by $h_{1}, \ldots, h_{m} \in \mathbb{R}[x]$ is defined as

$$
I=\left\langle h_{1}, \ldots h_{m}\right\rangle:=\left\{\sum_{j=1}^{m} u_{j} h_{j} \mid \quad u_{1}, \ldots, u_{m} \in \mathbb{R}[x]\right\}
$$

and the set $\left\{h_{1}, \ldots, h_{m}\right\}$ is then called a basis of $I$. By the finite basis theorem $[6, \S 2.5$, Thm. 4], every ideal in $\mathbb{R}[x]$ admits a finite basis. Given an ideal $I \subseteq \mathbb{R}[x]$, the algebraic variety of $I$ is the set

$$
V_{\mathbb{C}}(I)=\left\{v \in \mathbb{C}^{n} \mid h_{j}(v)=0 \forall j=1, \ldots, m\right\}
$$

of common complex zeros to all polynomials in $I$ and its real variety is

$$
V_{\mathbb{R}}(I):=V_{\mathbb{C}}(I) \cap \mathbb{R}^{n} .
$$

The ideal $I$ is said to be zero-dimensional when its complex variety $V_{\mathbb{C}}(I)$ is finite. The vanishing ideal of a subset $V \subseteq \mathbb{C}^{n}$ is the ideal

$$
\mathcal{I}(V):=\{f \in \mathbb{R}[x] \mid f(v)=0 \forall v \in V\} .
$$

For an ideal $I \subseteq \mathbb{R}[x]$, we may also define the ideal

$$
\sqrt{I}:=\left\{f \in \mathbb{R}[x] \mid \quad f^{m} \in I \text { for some } m \in \mathbb{N} \backslash\{0\}\right\},
$$

called the radical ideal of $I$, and the real radical ideal (or real ideal)

$$
\sqrt[\mathbb{R}]{I}:=\left\{p \in \mathbb{R}[x] \mid p^{2 m}+\sum_{j} q_{j}^{2} \in I \text { for some } q_{j} \in \mathbb{R}[x], m \in \mathbb{N} \backslash\{0\}\right\} .
$$

An ideal $I$ is said to be radical (resp., real radical) if $I=\sqrt{I}$ (resp., $I=\sqrt[\mathbb{R}]{I}$ ). For instance, the ideal $I=\left\langle x_{1}^{2}+x_{2}^{2}\right\rangle$ is not real radical since $x_{1}, x_{2} \in \sqrt[\mathbb{R}]{I} \backslash I$. As can be easily verified, $I$ is radical if and only if $p^{2} \in I$ implies $p \in I$, and $I$ is real radical if and only if $\sum_{i} p_{i}^{2} \in I$ implies $p_{i} \in I$ for all $i$. We have the following chains of inclusion:

$$
I \subseteq \sqrt{I} \subseteq \mathcal{I}\left(V_{\mathbb{C}}(I)\right), \quad I \subseteq \sqrt[\mathbb{R}]{I} \subseteq \mathcal{I}\left(V_{\mathbb{R}}(I)\right) .
$$

The relation between vanishing and (real) radical ideals is stated in the following two famous theorems:

Theorem 1. Let $I \subseteq \mathbb{R}[x]$ be an ideal.

(i) Hilbert's Nullstellensatz (see, e.g., [6, §4.1]) The radical ideal of I is equal to the vanishing ideal of its variety, i.e., $\sqrt{I}=\mathcal{I}\left(V_{\mathbb{C}}(I)\right)$.

(ii) Real Nullstellensatz (see, e.g., $[4, \S 4.1])$ The real radical ideal of I is equal to the vanishing ideal of its real variety, i.e., $\sqrt[\mathbb{R}]{I}=\mathcal{I}\left(V_{\mathbb{R}}(I)\right)$. 


\subsection{The eigenvalue method for complex roots}

The quotient space $\mathbb{R}[x] / I$. The quotient set $\mathbb{R}[x] / I$ consists of all cosets $[f]:=f+I=\{f+q \mid q \in I\}$ for $f \in \mathbb{R}[x]$, i.e. all equivalent classes of polynomials in $\mathbb{R}[x]$ modulo $I$. This quotient set $\mathbb{R}[x] / I$ is an algebra with addition $[f]+[g]:=[f+g]$, scalar multiplication $\lambda[f]:=[\lambda f]$ and multiplication $[f][g]:=[f g]$, for $\lambda \in \mathbb{R}, f, g \in \mathbb{R}[x]$. The following classical result relates the dimension of $\mathbb{R}[x] / I$ and the cardinality of the variety $V_{\mathbb{C}}(I)$ (see e.g. $[6,43]$ ).

Theorem 2. Let I be an ideal in $\mathbb{R}[x]$. Then,

$$
\left|V_{\mathbb{C}}(I)\right|<\infty \Longleftrightarrow \operatorname{dim} \mathbb{R}[x] / I<\infty .
$$

Moreover, $\left|V_{\mathbb{C}}(I)\right| \leq \operatorname{dim} \mathbb{R}[x] / I$, with equality if and only if $I$ is radical.

Assume that the number of complex roots is finite and set $N:=\operatorname{dim} \mathbb{R}[x] / I$, so that $\left|V_{\mathbb{C}}(I)\right| \leq N<\infty$. Consider a set $\mathcal{B}:=\left\{b_{1}, \ldots, b_{N}\right\} \subseteq \mathbb{R}[x]$ for which the cosets $\left[b_{1}\right], \ldots,\left[b_{N}\right]$ are pairwise distinct and $\left\{\left[b_{1}\right], \ldots,\left[b_{N}\right]\right\}$ is a (linear) basis of $\mathbb{R}[x] / I$. By abuse of language we also say that $\mathcal{B}$ itself is a basis of $\mathbb{R}[x] / I$. Then every $f \in \mathbb{R}[x]$ can be written in a unique way as $f=\sum_{i=1}^{N} c_{i} b_{i}+p$, where $c_{i} \in \mathbb{R}$ and $p \in I$. The polynomial

$$
\mathcal{N}_{\mathcal{B}}(f):=\sum_{i=1}^{N} c_{i} b_{i}
$$

is called the normal form of $f$ modulo $I$ with respect to the basis $\mathcal{B}$. In other words, we have the direct sum decomposition:

$$
\mathbb{R}[x]=\operatorname{Span}_{\mathbb{R}}(\mathcal{B}) \oplus I,
$$

and $\operatorname{Span}_{\mathbb{R}}(\mathcal{B})$ and $\mathbb{R}[x] / I$ are isomorphic vector spaces. We now introduce the eigenvalue method for computing all roots of a zero-dimensional ideal, which we first describe in the univariate case.

Computing roots with companion matrices. Consider first a univariate polynomial $p=x^{d}-a_{d-1} x^{d-1}-\ldots-a_{1} x-a_{0}$ and the ideal $I=\langle p\rangle$. Then the set $\mathcal{B}=\left\{1, x, \ldots, x^{d-1}\right\}$ is a basis of $\mathbb{R}[x] / I$. The following matrix

$$
\mathcal{X}:=\left(\begin{array}{cc}
0 & a_{0} \\
I_{d-1} & a
\end{array}\right) \quad \text { where } a=\left(a_{1}, \ldots, a_{d-1}\right)^{T},
$$

is known as the companion matrix of the polynomial $p$. One can easily verify that $\operatorname{det}(\mathcal{X}-x I)=(-1)^{d} p(x)$, so that the eigenvalues of $\mathcal{X}$ are precisely the roots of the polynomials $p$. Therefore the roots of a univariate polynomial can be found with an eigenvalue computation. Moreover, the columns of the companion matrix $\mathcal{X}$ correspond to the normal forms of the monomials in $x \mathcal{B}=\left\{x, x^{2}, \ldots, x^{d}\right\}$ modulo $I$ with respect to the basis $\mathcal{B}$. As we now see these facts extend naturally to the multivariate case. 
Given $h \in \mathbb{R}[x]$, we define the multiplication (by $h$ ) operator in $\mathbb{R}[x] / I$ as

$$
\begin{aligned}
\mathcal{X}_{h}: \mathbb{R}[x] / I & \longrightarrow \mathbb{R}[x] / I \\
{[f] } & \longmapsto \mathcal{X}_{h}([f]):=[h f],
\end{aligned}
$$

which can be represented by its matrix (again denoted $\mathcal{X}_{h}$ for simplicity) with respect to the basis $\mathcal{B}$ of $\mathbb{R}[x] / I$. Namely, if we set $\mathcal{N}_{\mathcal{B}}\left(h b_{j}\right):=\sum_{i=1}^{N} a_{i j} b_{i}$ (where $a_{i j} \in \mathbb{R}$ ), then the $j$ th column of $\mathcal{X}_{h}$ is the vector $\left(a_{i j}\right)_{i=1}^{N}$. Note also that, since $h b_{j}-\mathcal{N}_{\mathcal{B}}\left(h b_{j}\right) \in I$, polynomials in $I$ can be read directly from $\mathcal{X}_{h}$. This fact will play an important role for border bases (see Section 2.3). In the univariate case, when $I=\langle p\rangle$ and $h=x$, the multiplication matrix $\mathcal{X}_{x}$ is precisely the companion matrix $\mathcal{X}$ of $p$ introduced above. Throughout we also denote by $\mathcal{X}_{i}:=\mathcal{X}_{x_{i}}$ the multiplication operator by the variable $x_{i}$ in the multivariate case.

The following famous result (see e.g. [5, Chap. 2§4]) relates the eigenvalues of the multiplication operators in $\mathbb{R}[x] / I$ to the algebraic variety $V_{\mathbb{C}}(I)$. This result underlies the well known eigenvalue method, which plays a central role in many algorithms for complex root solving.

Theorem 3. (Stickelberger theorem) Let I be a zero-dimensional ideal in $\mathbb{R}[x]$, let $\mathcal{B}$ be a basis of $\mathbb{R}[x] / I$, and let $h \in \mathbb{R}[x]$. The eigenvalues of the multiplication operator $\mathcal{X}_{h}$ are the evaluations $h(v)$ of the polynomial $h$ at the points $v \in V_{\mathbb{C}}(I)$. Moreover, for all $v \in V_{\mathbb{C}}(I)$,

$$
\left(\mathcal{X}_{h}\right)^{T}[v]_{\mathcal{B}}=h(v)[v]_{\mathcal{B}},
$$

setting $[v]_{\mathcal{B}}=(b(v))_{b \in \mathcal{B}}$; that is, the vector $[v]_{\mathcal{B}}$ is a left eigenvector of the multiplication operator with eigenvalue $h(v)$.

Therefore the eigenvalues of the matrices $\mathcal{X}_{i}$ are the $i$ th coordinates of the points $v \in V_{\mathbb{C}}(I)$, which can be derived from the left eigenvectors $[v]_{\mathcal{B}}$. Practically, one can recover the roots from the left eigenvectors when the eigenspaces of $\mathcal{X}_{h}^{T}$ all have dimension one. This is the case when the values $h(v)\left(v \in V_{\mathbb{C}}(I)\right)$ are pairwise distinct (easy to achieve, e.g., if we choose $h$ to be a generic linear form) and when the ideal $I$ is radical (since the dimension of $\mathbb{R}[x] / I$ is then equal to the number of roots so that the vectors $[v]_{\mathcal{B}}\left(v \in V_{\mathbb{C}}(I)\right)$ form a complete basis of eigenvectors).

Summarizing, the task of solving a system of polynomial equations is reduced to a task of numerical linear algebra once a basis of $\mathbb{R}[x] / I$ and a normal form algorithm are available, as they permit the construction of the multiplication matrices $\mathcal{X}_{i}, \mathcal{X}_{h}$. Moreover, the roots $v \in V_{\mathbb{C}}(I)$ can be successfully constructed from the eigenvectors/eigenvalues of $\mathcal{X}_{h}$ when $I$ is radical and $h$ is generic. Our strategy for computing the real variety $V_{\mathbb{R}}(I)$ will be to compute a linear basis of the quotient space $\mathbb{R}[x] / \sqrt[\mathbb{R}]{I}$ and the corresponding multiplication matrices, so that we we can apply the eigenvalue method precisely in this setting of having a radical (even real radical) ideal. 
The number of (real) roots can be counted using Hermite's quadratic form:

$$
\begin{aligned}
S_{h}: \mathbb{R}[x] / I \times \mathbb{R}[x] / I & \rightarrow \mathbb{R} \\
([f],[g]) & \mapsto \operatorname{Tr}\left(\mathcal{X}_{f g h}\right) .
\end{aligned}
$$

Here, $\operatorname{Tr}\left(\mathcal{X}_{f g h}\right)$ is the trace of the multiplication (by the polynomial $f g h$ ) matrix. As $S_{h}$ is a symmetric matrix, all its eigenvalues are real. Denote by $\sigma_{+}\left(S_{h}\right)$ (resp., $\sigma_{-}\left(S_{h}\right)$ ) its number of positive (resp., negative) eigenvalues. The following classical result shows how to count the number of roots satisfying prescribed sign conditions (cf. e.g. [2]).

Theorem 4. Let $I \subseteq \mathbb{R}[x]$ be a zero-dimensional ideal and $h \in \mathbb{R}[x]$. Then,

$$
\begin{gathered}
\operatorname{rank} S_{h}=\left|\left\{v \in V_{\mathbb{C}}(I) \mid h(v) \neq 0\right\}\right|, \\
\sigma_{+}\left(S_{h}\right)-\sigma_{-}\left(S_{h}\right)=\left|\left\{v \in V_{\mathbb{R}}(I) \mid h(v)>0\right\}\right|-\left|\left\{v \in V_{\mathbb{R}}(I) \mid h(v)<0\right\}\right| .
\end{gathered}
$$

In particular, for the constant polynomial $h=1$,

$$
\operatorname{rank}\left(S_{1}\right)=\left|V_{\mathbb{C}}(I)\right| \text { and } \sigma_{+}\left(S_{1}\right)-\sigma_{-}\left(S_{1}\right)=\left|V_{\mathbb{R}}(I)\right| .
$$

\subsection{Border bases and normal forms}

The eigenvalue method for solving polynomial equations (described in the preceding section) requires the knowledge of a basis of $\mathbb{R}[x] / I$ and of an algorithm to compute the normal form of a polynomial with respect to this basis.

A well known basis of $\mathbb{R}[x] / I$ is the set of standard monomials with respect to some monomial ordering. The classical way to find standard monomials is to construct a Gröbner basis of $I$ (then the standard monomials are the monomials not divisible by any leading monomial of a polynomial in the Gröbner basis). Moreover, once a Gröbner basis is known, the normal form of a polynomial can be found via a polynomial division algorithm (see, e.g., [6, Chap. 1] for details). Other techniques have been proposed, producing more general bases which do not depend on a specific monomial ordering and often are numerically more stable. In particular, algorithms have been proposed for constructing border bases of $I$ leading to general (connected to 1) bases of $\mathbb{R}[x] / I$ (see [9, Chap. 4], [14], [29], [43]); these objects are introduced below. The moment matrix approach for computing real roots presented in this chapter leads naturally to the computation of such general bases.

Definition 1. Given a set $\mathcal{B}$ of monomials, define the new sets of monomials

$$
\mathcal{B}^{+}:=\mathcal{B} \cup \bigcup_{i=1}^{n} x_{i} \mathcal{B}=\mathcal{B} \cup\left\{x_{i} b \mid b \in \mathcal{B}, i=1, \ldots, n\right\}, \quad \partial \mathcal{B}=\mathcal{B}^{+} \backslash \mathcal{B},
$$

called, respectively, the one-degree prolongation of $\mathcal{B}$ and the border of $\mathcal{B}$. The set $\mathcal{B}$ is said to be connected to 1 if $1 \in \mathcal{B}$ and each $m \in \mathcal{B} \backslash\{1\}$ can be written as $m=x_{i_{1}} \ldots x_{i_{k}}$ with $x_{i_{1}}, x_{i_{1}} x_{i_{2}}, \ldots, x_{i_{1}} \cdots x_{i_{k}} \in \mathcal{B}$. Moreover, $\mathcal{B}$ is said to be stable by division if all divisors of $m \in \mathcal{B}$ also belong to $\mathcal{B}$. Obviously, $\mathcal{B}$ is connected to 1 if it is stable by division. 
Assume $\mathcal{B}$ is a set of monomials which is connected to 1 . For each border monomial $m \in \partial \mathcal{B}$, consider a polynomial $f_{m}$ of the form

$$
f_{m}:=m-r_{m}, \quad \text { where } r_{m} \in \operatorname{Span}_{\mathbb{R}}(\mathcal{B}) .
$$

The family $F:=\left\{f_{m} \mid m \in \partial \mathcal{B}\right\}$ is called a rewriting family for $\mathcal{B}$ in [30, 32]. Using $F$, one can express all border monomials in $\partial \mathcal{B}$ as linear combinations of monomials in $\mathcal{B}$ modulo the ideal $\langle F\rangle$. Moreover, the rewriting family $F$ can be used in a division algorithm to rewrite any polynomial $p \in \mathbb{R}[x]$ as

$$
p=r+\sum_{m \in \partial \mathcal{B}} u_{m} f_{m}, \quad \text { where } r \in \operatorname{Span}_{\mathbb{R}}(\mathcal{B}), u_{m} \in \mathbb{R}[x] .
$$

This expression is in general not unique, as it depends on the order in which the polynomials of $F$ are used throughout the division process.

Example 3. Let $\mathcal{B}=\left\{1, x_{1}, x_{2}\right\}$ with border set $\partial \mathcal{B}=\left\{x_{1}^{2}, x_{1} x_{2}, x_{2}^{2}\right\}$, and consider the rewriting family

$$
F=\left\{f_{x_{1}^{2}}=x_{1}^{2}+1, f_{x_{1} x_{2}}=x_{1} x_{2}-1, f_{x_{2}^{2}}=x_{2}^{2}+1\right\} .
$$

There are two possibilities to rewrite the polynomial $p=x_{1}^{2} x_{2}$. Either, first divide by $f_{x_{1} x_{2}}$ and obtain $p=x_{1}^{2} x_{2}=x_{1} f_{x_{1} x_{2}}+x_{1}$ with $r=x_{1}$, or first divide by $f_{x_{1}^{2}}$ and obtain $p=x_{1}^{2} x_{2}=x_{2} f_{x_{1}^{2}}-x_{2}$ with $r=-x_{2}$.

In view of (7), the set $\mathcal{B}$ spans the vector space $\mathbb{R}[x] /\langle F\rangle$, but is in general not linearly independent. Linear independence guaranties uniqueness of the decomposition (7) and, as Theorem 5 below shows, is equivalent to the commutativity of certain formal multiplication operators.

Consider the linear operator $\mathcal{X}_{i}: \operatorname{Span}_{\mathbb{R}}(\mathcal{B}) \rightarrow \operatorname{Span}_{\mathbb{R}}(\mathcal{B})$ defined using the rewriting family $F$, namely, for $b \in \mathcal{B}$,

$$
\mathcal{X}_{i}(b)= \begin{cases}x_{i} b & \text { if } x_{i} b \in \mathcal{B} \\ x_{i} b-f_{x_{i} b}=r_{x_{i} b} & \text { otherwise }\end{cases}
$$

and extend $\mathcal{X}_{i}$ to $\operatorname{Span}_{\mathbb{R}}(\mathcal{B})$ by linearity. Denote also by $\mathcal{X}_{i}$ the matrix of this linear operator, which can be seen as a formal multiplication (by $x_{i}$ ) matrix.

Theorem 5. [29] Let $F$ be a rewriting family for a set $\mathcal{B}$ of monomials connected to 1 , and consider the ideal $J:=\langle F\rangle$. The following conditions are equivalent:

(i) The formal multiplication matrices $\mathcal{X}_{1}, \ldots, \mathcal{X}_{n}$ commute pairwise.

(ii) The set $\mathcal{B}$ is a (linear) basis of $\mathbb{R}[x] / J$, i.e., $\mathbb{R}[x]=\operatorname{Span}_{\mathbb{R}}(\mathcal{B}) \oplus J$.

Then, the set $F$ is said to be a border basis of the ideal $J$, and the matrix $\mathcal{X}_{i}$ represents the multiplication operator by $x_{i}$ in $\mathbb{R}[x] / J$ with respect to $\mathcal{B}$. 
This theorem is the crucial tool for efficient root finding algorithms based on normal form reductions, which iteratively construct a system of polynomial equations giving a rewriting family corresponding to a commuting family of multiplication matrices (thus reducing the root finding problem to an eigenvalue computation, see [30]). We illustrate Theorem 5 on a small example.

Example 4 . Let $\mathcal{B}=\left\{1, x_{1}\right\}$ with border set $\partial \mathcal{B}=\left\{x_{2}, x_{1} x_{2}, x_{1}^{2}\right\}$, and consider the rewriting family

$$
F=\left\{f_{x_{1}^{2}}=x_{1}^{2}+1, f_{x_{1} x_{2}}=x_{1} x_{2}-1, f_{x_{2}}=x_{2}+x_{1}\right\} .
$$

As $x_{1} \in \mathcal{B}, x_{1}^{2}=f_{x_{1}^{2}}-1, x_{2}=f_{x_{2}}-x_{1}$, and $x_{2} x_{1}=f_{x_{1} x_{2}}+1$, we have

$$
\mathcal{X}_{1}=\left(\begin{array}{cc}
0 & -1 \\
1 & 0
\end{array}\right), \quad \mathcal{X}_{2}=\left(\begin{array}{cc}
0 & 1 \\
-1 & 0
\end{array}\right)
$$

As the formal multiplication matrices $\mathcal{X}_{1}, \mathcal{X}_{2}$ commute, we can conclude that $F$ is a border basis of $\langle F\rangle$ and $\mathbb{R}[x]=\operatorname{Span}_{\mathbb{R}}(\mathcal{B}) \oplus\langle F\rangle$.

\section{The moment method for real root finding}

We just saw that computing the complex roots of an ideal can be reduced to an eigenvalue computation. This technique applies only when the number of complex roots is finite, and involves matrices whose size is at least the number of complex roots. However, in most applications one is only interested in the real roots, whose number can be a very small fraction of the total number of roots. Therefore one needs a tool to isolate the real roots from the complex nonreal ones. As we briefly mentioned in the Introduction, a possible strategy is to add new polynomials from the real radical ideal to the original system to be solved. To find these polynomials in a systematic way we propose to work on the 'dual side', i.e. to consider linear forms $\Lambda$ on the polynomial ring $\mathbb{R}[x]$ or its subspaces $\mathbb{R}[x]_{t}$ of bounded degree. Indeed, it turns out that the kernel of such linear forms carries all information about the real radical ideal and the real variety when the linear form is assumed to satisfy some positivity condition. In this section we explain the method in detail and illustrate it on a few examples.

\subsection{Positive linear forms and real radical ideals}

Given a linear form $\Lambda \in \mathbb{R}[x]^{*}$, consider the quadratic form on $\mathbb{R}[x]$

$$
Q_{\Lambda}: f \in \mathbb{R}[x] \mapsto Q_{\Lambda}(f)=\Lambda\left(f^{2}\right) \in \mathbb{R},
$$

with kernel $\operatorname{Ker} Q_{\Lambda}:=\{f \in \mathbb{R}[x] \mid \Lambda(f g)=0 \forall g \in \mathbb{R}[x]\}$. 
Definition 2. (Positivity) $\Lambda \in \mathbb{R}[x]^{*}$ is said to be positive if $\Lambda\left(f^{2}\right) \geq 0$ for all $f \in \mathbb{R}[x]$, i.e., if the quadratic form $Q_{\Lambda}$ is positive semidefinite.

The following simple lemma provides the link to real radical polynomial ideals.

Lemma 1. [20, 26] Let $\Lambda \in \mathbb{R}[x]^{*}$. Then $\operatorname{Ker} Q_{\Lambda}$ is an ideal in $\mathbb{R}[x]$, which is real radical when $\Lambda$ is positive.

Proof. $\operatorname{Ker} Q_{\Lambda}$ is obviously an ideal, from its definition. Assume $\Lambda$ is positive. First we show that, for $p \in \mathbb{R}[x], \Lambda\left(p^{2}\right)=0$ implies $\Lambda(p)=0$. Indeed, if $\Lambda\left(p^{2}\right)=0$ then, for any scalar $t \in \mathbb{R}$, we have:

$$
0 \leq \Lambda\left((p+t)^{2}\right)=\Lambda\left(p^{2}\right)+2 t \Lambda(p)+t^{2} \Lambda(1)=t(2 \Lambda(p)+t \Lambda(1)),
$$

which implies $\Lambda(p)=0$. Assume now $\sum_{i} p_{i}^{2} \in \operatorname{Ker} Q_{\Lambda}$ for some $p_{i} \in \mathbb{R}[x]$; we show $p_{i} \in \operatorname{Ker} Q_{\Lambda}$. For any $g \in \mathbb{R}[x]$, we have $0=\Lambda\left(g^{2}\left(\sum_{i} p_{i}^{2}\right)\right)=\sum_{i} \Lambda\left(p_{i}^{2} g^{2}\right)$ which, as $\Lambda\left(p_{i}^{2} g^{2}\right) \geq 0$, implies $\Lambda\left(p_{i}^{2} g^{2}\right)=0$. By the above, this in turn implies $\Lambda\left(p_{i} g\right)=0$, thus showing $p_{i} \in \operatorname{Ker} Q_{\Lambda}$. Therefore, $\operatorname{Ker} Q_{\Lambda}$ is real radical.

We now introduce moment matrices, which permit to reformulate positivity of $\Lambda$ in terms of positive semidefiniteness of an associated matrix $M(\Lambda)$.

Definition 3. (Moment matrix) A symmetric matrix $M=\left(M_{\alpha, \beta}\right)$ indexed by $\mathbb{N}^{n}$ is said to be a moment matrix (or a generalized Hankel matrix) if its $(\alpha, \beta)$-entry depends only on the sum $\alpha+\beta$ of the indices. Given $\Lambda \in \mathbb{R}[x]^{*}$, the matrix

$$
M(\Lambda):=\left(\Lambda\left(x^{\alpha} x^{\beta}\right)\right)_{\alpha, \beta \in \mathbb{N}^{n}}
$$

is called the moment matrix of $\Lambda$.

If $y \in \mathbb{R}^{\mathbb{N}^{n}}$ is the coefficient series of $\Lambda \in \mathbb{R}[x]^{*}$, i.e., $\Lambda=\sum_{\alpha} y_{\alpha} \partial_{0}^{\alpha}$, then its moment matrix $M(y)=\left(y_{\alpha+\beta}\right)_{\alpha, \beta \in \mathbb{N}^{n}}$ coincides with the moment matrix $M(\Lambda)$ of $\Lambda$. These two definitions are obviously equivalent and, depending on the context, it is more convenient to use $M(y)$ or $M(\Lambda)$.

Note that $Q_{\Lambda}(p)=\Lambda\left(p^{2}\right)=\operatorname{vec}(p)^{T} M(\Lambda) \operatorname{vec}(p)$ for all $p \in \mathbb{R}[x]$. Hence, $M(\Lambda)$ is the matrix of the quadratic form $Q_{\Lambda}$ in the monomial base, and $\Lambda$ is positive if and only if $M(\Lambda) \succeq 0$.

Moreover, a polynomial $p$ belongs to the kernel of $Q_{\Lambda}$ if and only if its coefficient vector belongs to $\operatorname{Ker} M(\Lambda)$. Throughout we identify polynomials $p=\sum_{\alpha} p_{\alpha} x^{\alpha}$ with their coefficient vectors $\operatorname{vec}(p)=\left(p_{\alpha}\right)_{\alpha}$ and thus $\operatorname{Ker} Q_{\Lambda}$ with $\operatorname{Ker} M(\Lambda)$. Hence we view $\operatorname{Ker} M(\Lambda)$ as a set of polynomials. By Lemma $1, \operatorname{Ker} M(\Lambda)$ is an ideal of $\mathbb{R}[x]$, which is real radical when $M(\Lambda) \succeq 0$. Moreover, the next lemma shows that $\operatorname{Ker} M(\Lambda)$ is a zero-dimensional ideal precisely when the matrix $M(\Lambda)$ has finite rank.

Example 5. For $n=2$, consider the linear form $\Lambda \in \mathbb{R}[x]^{*}$ defined by $\Lambda(1)=$ $\Lambda\left(x_{1}^{2}\right)=1$ and $\Lambda\left(x_{1}^{\alpha_{1}} x_{2}^{\alpha_{2}}\right)=0$ for all other monomials. Then $\Lambda$ is positive, $\operatorname{rank} M(\Lambda)=2$ and the kernel of $M(\Lambda)$ is the ideal $\left\langle x_{2}, 1-x_{1}^{2}\right\rangle$. 
Lemma 2. Let $\Lambda \in \mathbb{R}[x]^{*}$ and let $\mathcal{B}$ be a set of monomials. Then, $\mathcal{B}$ indexes a maximal linearly independent set of columns of $M(\Lambda)$ if and only if $\mathcal{B}$ corresponds to a basis of $\mathbb{R}[x] / \operatorname{Ker} M(\Lambda)$. That is,

$$
\operatorname{rank} M(\Lambda)=\operatorname{dim} \mathbb{R}[x] / \operatorname{Ker} M(\Lambda) .
$$

Next we collect some properties of the moment matrix of evaluations at points of $\mathbb{R}^{n}$.

Lemma 3. If $\Lambda=\Lambda_{v}$ is the evaluation at $v \in \mathbb{R}^{n}$, then $M\left(\Lambda_{v}\right)=[v]_{\infty}[v]_{\infty}^{T}$ has rank 1 and its kernel is $\mathcal{I}(v)$, the vanishing ideal of $v$. More generally, if $\Lambda$ is a conic combination of evaluations at real points, say $\Lambda=\sum_{i=1}^{r} \lambda_{i} \Lambda_{v_{i}}$ where $\lambda_{i}>0$ and $v_{i} \in \mathbb{R}^{n}$ are pairwise distinct, then $M(\Lambda)=\sum_{i=1}^{r} \lambda_{i}\left[v_{i}\right]_{\infty}\left[v_{i}\right]_{\infty}^{T}$ has rank $r$ and its kernel is $\mathcal{I}\left(v_{1}, \ldots, v_{r}\right)$, the vanishing ideal of the $v_{i}$ 's.

The following theorem of Curto and Fialkow [7] shows that any positive linear form $\Lambda$ with a finite rank moment matrix is a conic combination of evaluations at real points. In other words, it shows that the implication of Lemma 3 holds as an equivalence. This result will play a crucial role in our approach. We give a proof, based on [20], although some details are simplified.

Theorem 6. (Finite rank moment matrix theorem) [7] Assume that $\Lambda \in \mathbb{R}[x]^{*}$ is positive with $\operatorname{rank} M(\Lambda)=: r<\infty$. Then, $\Lambda=\sum_{i=1}^{r} \lambda_{i} \Lambda_{v_{i}}$ for some distinct $v_{1}, \ldots, v_{r} \in \mathbb{R}^{n}$ and some scalars $\lambda_{i}>0$. Moreover, $\left\{v_{1}, \ldots, v_{r}\right\}=V_{\mathbb{C}}(\operatorname{Ker} M(\Lambda))$.

Proof. By Lemma $1, J:=\operatorname{Ker} M(\Lambda)$ is a real radical ideal and, by Lemma 2 (combined with Theorem 2), $J$ is zero-dimensional with $\operatorname{dim} \mathbb{R}[x] / J=r$. Therefore, $\left|V_{\mathbb{C}}(J)\right|=r$ and $V_{\mathbb{C}}(J) \subseteq \mathbb{R}^{n}$. Say,

$$
V_{\mathbb{C}}(J)=\left\{v_{1}, \ldots, v_{r}\right\} \subseteq \mathbb{R}^{n}
$$

so that $J=\mathcal{I}\left(v_{1}, \ldots, v_{r}\right)$ is the vanishing ideal of the $v_{i}$ 's. Let $p_{1}, \ldots, p_{r}$ be interpolation polynomials at $v_{1}, \ldots, v_{r}$, respectively, that is, $p_{i}\left(v_{j}\right)=1$ if $i=j$ and 0 otherwise. We first claim:

The set $\left\{p_{1}, \ldots, p_{r}\right\}$ forms a basis of the quotient space $\mathbb{R}[x] / J$.

Indeed if, for some scalars $\lambda_{i}$, the polynomial $\sum_{i=1}^{r} \lambda_{i} p_{i}$ vanishes at all $v_{i}$ 's, then $\lambda_{i}=0$ for all $i$. Hence the set $\left\{p_{1}, \ldots, p_{r}\right\}$ is linearly independent in $\mathbb{R}[x] / J$ and thus it is a basis, since $r=\operatorname{dim} \mathbb{R}[x] / J$. Consider the linear form

$$
\Lambda^{\prime}:=\sum_{i=1}^{r} \Lambda\left(p_{i}^{2}\right) \Lambda_{v_{i}} .
$$

We claim that $\Lambda=\Lambda^{\prime}$. As both $\Lambda$ and $\Lambda^{\prime}$ vanish on the ideal $J$, it suffices to show that $\Lambda$ and $\Lambda^{\prime}$ take the same values at all members of the basis $\left\{p_{1}, \ldots, p_{r}\right\}$ of $\mathbb{R}[x] / J$. Indeed, $\Lambda^{\prime}\left(p_{j}\right)=\Lambda\left(p_{j}^{2}\right)$ (since $p_{j}\left(v_{i}\right)=\delta_{i, j}$ ), and $\Lambda\left(p_{j}\right)=\Lambda\left(p_{j}^{2}\right)$ as well $\left(\right.$ since $\left.p_{j}-p_{j}^{2} \in J\right)$. 
Example 6. Consider the linear form $\Lambda=\frac{1}{2} \Lambda_{(0,0)}+\frac{1}{2} \Lambda_{(1,2)} \in \mathbb{R}[x]^{*}$, with moment matrix (indexed by $1, x_{1}, x_{2}, x_{1}^{2}, \ldots$ ):

$$
M(\Lambda)=\left(\begin{array}{ccccc}
1 & \frac{1}{2} & 1 & \frac{1}{2} & \cdots \\
\frac{1}{2} & \frac{1}{2} & 1 & \frac{1}{2} & \cdots \\
1 & 1 & 2 & 1 & \cdots \\
\frac{1}{2} & \frac{1}{2} & 1 & \frac{1}{2} & \cdots \\
\vdots & \vdots & \vdots & \vdots & \ddots
\end{array}\right)=\left.\frac{1}{2}\left[v_{1}\right]_{\infty}\left[v_{1}\right]_{\infty}^{T}\right|_{v_{1}=(0,0)}+\left.\frac{1}{2}\left[v_{2}\right]_{\infty}\left[v_{2}\right]_{\infty}^{T}\right|_{v_{2}=(1,2)}
$$

Note e.g. that the 2 nd and 4 th columns of $M(\Lambda)$ coincide, yielding the polynomial $g_{1}=-x_{1}+x_{1}^{2}$ in the kernel of $M(\Lambda)$. In fact, the polynomials $g_{1}$, $g_{2}=-2 x_{1}+x_{2}, g_{3}=-2 x_{1}+x_{1} x_{2}$ provide a basis of the real radical ideal $\operatorname{Ker} M(\Lambda)$, whose variety is $V_{\mathbb{C}}(\operatorname{Ker} M(\Lambda))=\{(0,0),(1,2)\} \subseteq \mathbb{R}^{2}$.

As background information we mention (without proof) the following characterization for the linear forms $\Lambda \in \mathbb{R}[x]^{*}$ with a finite rank moment matrix. When positivity is dropped, the evaluations at points $v \in V_{\mathbb{C}}(\Lambda)$ do not suffice, one also needs the more general differential operators $\partial_{v}^{\alpha}$ (defined in (4)).

Theorem 7. (see [9, Thm 2.2.7], [10, Chap. 7]) Let $\Lambda \in \mathbb{R}[x]^{*}$ satisfying $\operatorname{rank} M(\Lambda)<\infty$. Say, $V_{\mathbb{C}}(\operatorname{Ker} M(\Lambda))=\left\{v_{1}, \ldots, v_{r}\right\}$, so that $r \leq \operatorname{rank} M(\Lambda)$. Then,

$$
\Lambda=\sum_{i=1}^{r} \sum_{\alpha \in A_{i}} a_{\alpha, i} \partial_{v_{i}}^{\alpha}
$$

where $A_{i} \subseteq \mathbb{N}^{n}$ are finite and $a_{\alpha, i} \in \mathbb{R} \backslash\{0\}$. Moreover, Ker $M(\Lambda)$ is radical if and only if

$$
\Lambda=\sum_{i=1}^{r} a_{i} \Lambda_{v_{i}}
$$

where $a_{i} \neq 0$ (i.e., $A_{i}=\{0\}$ for all $i$ ). Furthermore, $\operatorname{Ker} M(\Lambda)$ is real radical precisely when (8) holds with $\left\{v_{1}, \ldots, v_{r}\right\} \subseteq \mathbb{R}^{n}$.

Excursion: Why is $M(\Lambda)$ called a moment matrix? We briefly recall how the matrices $M(\Lambda)$ arise naturally in the context of the classical moment problem in mathematics (cf. e.g. [1]). Given a finite positive Borel measure $\mu$ on $\mathbb{R}^{n}$, the quantity

$$
\int_{\mathbb{R}^{n}} x^{\alpha} d \mu
$$

is called its moment of order $\alpha \in \mathbb{N}^{n}$, and the sequence $y_{\mu}=\left(\int x^{\alpha} d \mu\right)_{\alpha \in \mathbb{N}^{n}}$ is called its moment sequence. The moment problem asks to characterize the sequences $y \in \mathbb{R}^{\mathbb{N}^{n}}$ that are the sequence of moments of some finite positive Borel measure on (some subset of) $\mathbb{R}^{n}$ or, equivalently, to characterize the linear forms $\Lambda \in \mathbb{R}[x]^{*}$ of the form 


$$
\Lambda=\Lambda_{\mu}(p):=\int p(x) d \mu \text { for } p \in \mathbb{R}[x]
$$

When (9) holds, $\mu$ is called a representing measure for $\Lambda$. A well known result of Haviland [11] claims that $\Lambda$ has a representing measure if and only if $\Lambda(p) \geq 0$ for all polynomials $p$ that are nonnegative on $\mathbb{R}^{n}$. However, except in some exceptional cases $^{3}$ no characterization is known for the nonnegative polynomials on $\mathbb{R}^{n}$. Yet we find the following well known necessary condition: If $\Lambda$ has a representing measure, then $\Lambda\left(p^{2}\right) \geq 0$ for all polynomials $p$, i.e., $\Lambda$ is positive, which is characterized by $M(\Lambda) \succeq 0$.

Positivity of $\Lambda$ is in general only a necessary condition for existence of a representing measure. However, the above result of Curto and Fialkow (Theorem 6 ) shows equivalence in the case when $M(\Lambda)$ has finite rank, in which case the measure $\mu$ is finite atomic with support $V_{\mathbb{C}}(\operatorname{Ker} M(\Lambda))$.

When $\mu=\delta_{v}$ is the Dirac measure at a point $v \in \mathbb{R}^{n}$, its moment sequence is $y_{\mu}=[v]_{\infty}$ with corresponding linear form $\Lambda_{\mu}=\Lambda_{v}$, the evaluation at $v$. More generally, when $\mu$ is finitely atomic, i.e., of the form $\mu=\sum_{i=1}^{r} \lambda_{i} \delta_{v_{i}}$ with finite support $\left\{v_{1}, \ldots, v_{r}\right\} \subseteq \mathbb{R}^{n}$, then its moment sequence is $y_{\mu}=$ $\sum_{i=1}^{r} \lambda_{i}\left[v_{i}\right]_{\infty}$ with corresponding linear form $\Lambda_{\mu}=\sum_{i=1}^{r} \lambda_{i} \Lambda_{v_{i}}$.

Characterizing real radical ideals using positive linear forms on $\mathbb{R}[x]$. We now combine the above results to obtain a semidefinite characterization of real radical ideals using positive linear forms. For this define the convex set

$$
\mathcal{K}=\left\{\Lambda \in \mathbb{R}[x]^{*} \mid \Lambda(1)=1, M(\Lambda) \succeq 0 \text { and } \Lambda(p)=0 \forall p \in I\right\}
$$

For any $\Lambda \in \mathcal{K}, \operatorname{Ker} M(\Lambda)$ is a real radical ideal, which contains $I$ and thus its real radical $\sqrt[\mathbb{R}]{I}$. This implies:

$$
\operatorname{dim} \mathbb{R}[x] / \operatorname{Ker} M(\Lambda) \leq \operatorname{dim} \mathbb{R}[x] / \sqrt[\mathbb{R}]{I}
$$

When the real variety $V_{\mathbb{R}}(I)$ is finite, $\mathbb{R}[x] / \sqrt[\mathbb{R}]{I}$ has finite dimension as a vector space, equal to $\left|V_{\mathbb{R}}(I)\right|$, and thus $\operatorname{Ker} M(\Lambda)$ is zero-dimensional with

$$
\operatorname{rank} M(\Lambda)=\operatorname{dim} \mathbb{R}[x] / \operatorname{Ker} M(\Lambda) \leq \operatorname{dim} \mathbb{R}[x] / \sqrt[\mathbb{R}]{I}=\left|V_{\mathbb{R}}(I)\right|
$$

(using Lemma 2 for the left most equality). Equality: $\operatorname{rank} M(\Lambda)=\left|V_{\mathbb{R}}(I)\right|$ holds, for instance, for the element $\Lambda=\frac{1}{\left|V_{\mathbb{R}}(I)\right|} \sum_{v \in V_{\mathbb{R}}(I)} \Lambda_{v}$ of $\mathcal{K}$. This fact motivates the following definition:

\footnotetext{
${ }^{3}$ A celebrated result of Hilbert (cf. e.g. [2]) shows that there are three sets of parameters $(n, d)$ for which the following equivalence holds: For any polynomial $p$ in $n$ variables and degree $2 d, p$ is nonnegative on $\mathbb{R}^{n}$ if and only if $p$ can be written as a sum of squares of polynomials. These parameters are $(n=1, d)$ (univariate polynomials), $(n, d=1)$ (quadratic polynomials), and ( $n=3, d=2$ ) (ternary quartic polynomials). In all other cases there are polynomials that are nonnegative on $\mathbb{R}^{n}$ but cannot be written as a sum of squares of polynomials.
} 
Definition 4. (Generic linear forms) Let $\mathcal{K}$ be defined as in (10) and assume $\left|V_{\mathbb{R}}(I)\right|<\infty$. A linear form $\Lambda \in \mathcal{K}$ is said to be generic if $M(\Lambda)$ has maximum rank, i.e., if $\operatorname{rank} M(\Lambda)=\left|V_{\mathbb{R}}(I)\right|$.

A simple geometric property of positive semidefinite matrices yields the following equivalent definition for generic elements of $\mathcal{K}$. This is in fact the key tool used in [17] for computing the real radical ideal $\sqrt[\mathbb{R}]{I}$.

Lemma 4. Assume $\left|V_{\mathbb{R}}(I)\right|<\infty$. An element $\Lambda \in \mathcal{K}$ is generic if and only if $\operatorname{Ker} M(\Lambda) \subseteq \operatorname{Ker} M\left(\Lambda^{\prime}\right)$ for all $\Lambda^{\prime} \in \mathcal{K}$. Moreover, $\operatorname{Ker} M(\Lambda)=\sqrt[\mathbb{R}]{I}$ for all generic $\Lambda \in \mathcal{K}$.

Proof. Assume first that $\operatorname{rank} M(\Lambda)=r$, with $r=\left|V_{\mathbb{R}}(I)\right|$ and $V_{\mathbb{R}}(I)=$ $\left\{v_{1}, \ldots, v_{r}\right\}$. As $\Lambda+\Lambda^{\prime} \in \mathcal{K}$ for $\Lambda^{\prime} \in \mathcal{K}$, we have

$$
\operatorname{Ker} M\left(\Lambda+\Lambda^{\prime}\right)=\operatorname{Ker} M(\Lambda) \cap \operatorname{Ker} M\left(\Lambda^{\prime}\right) \subseteq \operatorname{Ker} M(\Lambda),
$$

implying $r \geq \operatorname{rank} M\left(\Lambda+\Lambda^{\prime}\right) \geq \operatorname{rank} M(\Lambda)$. Hence equality holds throughout which implies $\operatorname{Ker} M(\Lambda)=\operatorname{Ker} M(\Lambda) \cap \operatorname{Ker} M\left(\Lambda^{\prime}\right) \subseteq \operatorname{Ker} M\left(\Lambda^{\prime}\right)$.

Conversely, assume $\operatorname{Ker} M(\Lambda) \subseteq \operatorname{Ker} M\left(\Lambda^{\prime}\right)$ for all $\Lambda^{\prime} \in \mathcal{K}$. Consider $\Lambda^{\prime}=\sum_{i=1}^{r} \Lambda_{v_{i}} \in \mathcal{K}$ whose kernel is $\mathcal{I}\left(v_{1}, \ldots, v_{r}\right)$. This implies $\operatorname{Ker} M(\Lambda) \subseteq$ $\mathcal{I}\left(v_{1}, \ldots, v_{r}\right)$ and thus

$$
\operatorname{rank} M(\Lambda)=\operatorname{dim} \mathbb{R}[x] / \operatorname{Ker} M(\Lambda) \geq \operatorname{dim} \mathbb{R}[x] / \mathcal{I}\left(v_{1}, \ldots, v_{r}\right)=r .
$$

Hence, $\operatorname{rank} M(\Lambda)=r$ and $\operatorname{Ker} M(\Lambda)=\mathcal{I}\left(v_{1}, \ldots, v_{r}\right)=\sqrt[\mathbb{R}]{I}$ (using the Real Nullstellensatz, Theorem 1 (ii), for the last equality).

Example 7 (Example 6 cont.). Consider the set $\mathcal{K}$ corresponding to the ideal $I=\left\langle h_{1}, h_{2}, h_{3}\right\rangle \subseteq \mathbb{R}\left[x_{1}, x_{2}\right]$, where

$$
h_{1}=x_{2}^{4} x_{1}+3 x_{1}^{3}-x_{2}^{4}-3 x_{1}^{2}, h_{2}=x_{1}^{2} x_{2}-2 x_{1}^{2}, h_{3}=2 x_{2}^{4} x_{1}-x_{1}^{3}-2 x_{2}^{4}+x_{1}^{2} .
$$

Then, $\Lambda=\frac{1}{2} \Lambda_{(0,0)}+\frac{1}{2} \Lambda_{(1,2)}$ is a generic element of $\mathcal{K}$. Thus the real radical ideal of $I$ is $\sqrt[\mathbb{R}]{I}=\operatorname{Ker} M(\Lambda)=\left\langle g_{1}, g_{2}, g_{3}\right\rangle$, with $g_{1}, g_{2}, g_{3}$ as in Example 6 .

\subsection{Truncated positive linear forms and real radical ideals}

In view of the results in the previous section (in particular, Lemmas 2 and 4), the task of finding the real radical ideal $\sqrt[\mathbb{R}]{I}$ as well as a linear basis of the quotient space $\mathbb{R}[x] / \sqrt[\mathbb{R}]{I}$ can be reduced to finding a generic linear form $\Lambda$ in the set $\mathcal{K}$ (defined in (10)). In order to be able to deal with such linear forms computationally, we will work with linear forms on finite dimensional subspaces $\mathbb{R}[x]_{s}$ of the polynomial ring. Given $\Lambda \in\left(\mathbb{R}[x]_{2 s}\right)^{*}$, we can define the quadratic form:

$$
Q_{\Lambda}: f \in \mathbb{R}[x]_{s} \mapsto Q_{\Lambda}(f)=\Lambda\left(f^{2}\right),
$$


whose matrix

$$
M_{s}(\Lambda)=\left(\Lambda\left(x^{\alpha} x^{\beta}\right)\right)_{\alpha, \beta \in \mathbb{N}_{s}^{n}}
$$

in the monomial basis of $\mathbb{R}[x]_{s}$ is called the truncated moment matrix of order $s$ of $\Lambda$. Thus $\Lambda$ is positive (i.e., $\Lambda\left(f^{2}\right) \geq 0 \forall f \in \mathbb{R}[x]_{s}$ ) if and only if $M_{s}(\Lambda) \succeq 0$. Again we identify the kernels of $Q_{\Lambda}$ and of $M_{s}(\Lambda)$ (by identifying polynomials with their coefficient sequences) and view $\operatorname{Ker} M_{s}(\Lambda)$ as a subset of $\mathbb{R}[x]_{s}$.

Flat extensions of moment matrices. We now present the following crucial result of Curto and Fialkow [7] for flat extensions of moment matrices.

Theorem 8. (Flat extension theorem) ([7], see also [21]) Let $\Lambda \in\left(\mathbb{R}[x]_{2 s}\right)^{\text {* }}$ and assume that $M_{s}(\Lambda)$ is a flat extension of $M_{s-1}(\Lambda)$, i.e.,

$$
\operatorname{rank} M_{s}(\Lambda)=\operatorname{rank} M_{s-1}(\Lambda) .
$$

Then one can extend (uniquely) $\Lambda$ to $\tilde{\Lambda} \in\left(\mathbb{R}[x]_{2 s+2}\right)^{*}$ in such a way that $M_{s+1}(\tilde{\Lambda})$ is a flat extension of $M_{s}(\Lambda) ;$ thus $\operatorname{rank} M_{s+1}(\tilde{\Lambda})=\operatorname{rank} M_{s}(\Lambda)$.

The proof is elementary and relies on the following lemma showing that the kernel of a truncated moment matrix behaves like a 'truncated ideal'.

Lemma 5. Let $\Lambda \in\left(\mathbb{R}[x]_{2 s}\right)^{*}$ and $f, g \in \mathbb{R}[x]$ with $f \in \operatorname{Ker} M_{s}(\Lambda)$.

(i) Assume $\operatorname{rank} M_{s}(\Lambda)=\operatorname{rank} M_{s-1}(\Lambda)$. Then $\operatorname{Ker} M_{s-1}(\Lambda) \subseteq \operatorname{Ker} M_{s}(\Lambda)$ and $f g \in \operatorname{Ker} M_{s}(\Lambda)$ if $\operatorname{deg}(f g) \leq s$.

(ii) Assume $M_{s}(\Lambda) \succeq 0$. Then $\operatorname{Ker} M_{s-1}(\Lambda) \subseteq \operatorname{Ker} M_{s}(\Lambda)$ and $f g \in \operatorname{Ker} M_{s}(\Lambda)$ if $\operatorname{deg}(f g) \leq s-1$.

Indeed, using property (11) and Lemma 5 (i), we see that for every monomial $m$ of degree $s$, there exists a polynomial of the form $f_{m}=m+r_{m} \in \operatorname{Ker} M_{s}(\Lambda)$, where $r_{m} \in \mathbb{R}[x]_{s-1}$. If an extension $\tilde{\Lambda}$ exists, then all the polynomials $f_{m}, x_{i} f_{m}$ must lie in the kernel of $M_{s+1}(\tilde{\Lambda})$ and they can be used to determine the unknown columns of $M_{s+1}(\tilde{\Lambda})$ indexed by monomials of degree $s+1$. The main work consists of verifying the consistency of this construction; namely, that the matrix constructed in this way is a moment matrix, i.e. that its $(\alpha, \beta)$ th entry depends only on the sum $\alpha+\beta$ when $|\alpha+\beta|=2 s+1,2 s+2$.

The flat extension theorem plays a crucial role in the moment matrix approach as it allows to deduce information about the infinite moment matrix $M(\Lambda)$ from its finite section $M_{s}(\Lambda)$.

Theorem 9. [17] Let $\Lambda \in\left(\mathbb{R}[x]_{2 s}\right)^{*}$ and assume that (11) holds. Then one can extend $\Lambda$ to $\tilde{\Lambda} \in \mathbb{R}[x]^{*}$ in such a way that $M(\tilde{\Lambda})$ is a flat extension of $M_{s}(\Lambda)$, and the ideal $\operatorname{Ker} M(\tilde{\Lambda})$ is generated by the polynomials in $\operatorname{Ker} M_{s}(\Lambda)$, i.e.,

$$
\operatorname{rank} M(\tilde{\Lambda})=\operatorname{rank} M_{s}(\Lambda) \text { and } \operatorname{Ker} M(\tilde{\Lambda})=\left\langle\operatorname{Ker} M_{s}(\Lambda)\right\rangle .
$$

Moreover, any monomial set $\mathcal{B}$ indexing a basis of the column space of $M_{s-1}(\Lambda)$ is a basis of the quotient space $\mathbb{R}[x] / \operatorname{Ker} M(\tilde{\Lambda})$. If, moreover, $M_{s}(\Lambda) \succeq 0$, then the ideal $\left\langle\operatorname{Ker} M_{s}(\Lambda)\right\rangle$ is real radical and $\Lambda$ is of the form $\Lambda=\sum_{i=1}^{r} \lambda_{i} \Lambda_{v_{i}}$, where $\lambda_{i}>0$ and $\left\{v_{1}, \ldots, v_{r}\right\}=V_{\mathbb{C}}\left(\operatorname{Ker} M_{s}(\Lambda)\right) \subseteq \mathbb{R}^{n}$. 
Proof. The existence of $\tilde{\Lambda}$ follows by applying iteratively Theorem 8 and the inclusion $\left\langle\operatorname{Ker} M_{s}(\Lambda)\right\rangle \subseteq \operatorname{Ker} M(\tilde{\Lambda})$ follows using Lemma 5 (i). If $\mathcal{B}$ is a set of monomials indexing a column basis of $M_{s-1}(\Lambda)$, then $\mathcal{B}$ is also a column basis of $M(\tilde{\Lambda})$ and thus a basis of $\mathbb{R}[x] / \operatorname{Ker} M(\tilde{\Lambda})$ (by Lemma 2). One can verify the direct sum decomposition $\mathbb{R}[x]=\operatorname{Span}_{\mathbb{R}}(\mathcal{B}) \oplus\left\langle\operatorname{Ker} M_{s}(\Lambda)\right\rangle$, which implies $\operatorname{Ker} M(\tilde{\Lambda})=\left\langle\operatorname{Ker} M_{s}(\Lambda)\right\rangle$. Finally, as $\tilde{\Lambda}$ is a flat extension of $\Lambda, M_{s}(\Lambda) \succeq$ 0 implies $M(\tilde{\Lambda}) \succeq 0$, so that $\left\langle\operatorname{Ker} M_{s}(\Lambda)\right\rangle=\operatorname{Ker} M(\tilde{\Lambda})$ is real radical (by Lemma 1). The final statement follows directly by applying Theorem 6 to $\tilde{\Lambda}$.

Example 8 (Example 6 cont.). Consider the linear form $\Lambda \in \mathbb{R}[x]^{*}$ in Example 6 . Recall that $\operatorname{Ker} M(\Lambda)$ is generated by $g_{1}, g_{2}, g_{3} \in \mathbb{R}[x]_{2}$. First note that these polynomials imply the rank condition: $\operatorname{rank} M_{2}(\Lambda)=\operatorname{rank} M_{1}(\Lambda)$ and thus permit to construct $M_{2}(\Lambda)$ from $M_{1}(\Lambda)$. Moreover, they permit to recover the infinite matrix $M(\Lambda)$ from its submatrix $M_{1}(\Lambda)$. For instance, since $x_{1}^{2} x_{2}=x_{2}\left(x_{1}+g_{1}\right)=2 x_{1}+g_{3}+g_{1} x_{2}$ and $g_{1}, g_{2}, g_{3} \in \operatorname{Ker} M_{2}(\Lambda) \subseteq \operatorname{Ker} M(\Lambda)$, we deduce that the column of $M(\Lambda)$ indexed by $x_{1}^{2} x_{2}$ is equal to twice its column indexed by $x_{1}$. Using the fact that $\operatorname{Ker} M(\Lambda)=\left\langle\operatorname{Ker} M_{2}(\Lambda)\right\rangle$, we can analogously define iteratively all columns of $M(\Lambda)$.

Computing real radical ideals using truncated positive linear forms on $\mathbb{R}[x]_{t}$. We saw above how to use positive linear forms on $\mathbb{R}[x]$ to characterize the real radical ideal $\sqrt[\mathbb{R}]{I}$. We now combine this characterization with the above results about flat extensions of truncated moment matrices to obtain a practical algorithm for computing $\sqrt[\mathbb{R}]{I}$ operating on finite dimensional subspaces $\mathbb{R}[x]_{t} \subseteq \mathbb{R}[x]$ only. As before $I=\left\langle h_{1}, \ldots, h_{m}\right\rangle$ is the ideal generated by the polynomial equations $h_{i}$ to be solved. For $t \in \mathbb{N}$, define the set

$$
\mathcal{H}_{t}=\left\{h_{i} x^{\alpha}|i=1, \ldots, m,| \alpha \mid \leq t-\operatorname{deg}\left(h_{i}\right)\right\}
$$

of prolongations up to degree $t$ of the polynomials $h_{i}$, and the truncated analogue of the set $\mathcal{K}$ :

$$
\mathcal{K}_{t}=\left\{\Lambda \in\left(\mathbb{R}[x]_{t}\right)^{*} \mid \Lambda(1)=1, M_{\lfloor t / 2\rfloor}(\Lambda) \succeq 0 \text { and } \Lambda(f)=0 \forall f \in \mathcal{H}_{t}\right\} .
$$

Note that the constraint: $\Lambda(f)=0 \forall f \in \mathcal{H}_{t}$ (i.e., $\Lambda \in \mathcal{H}_{t}^{\perp}$ ) corresponds to the constraint (2) of Section 1.2. As the convex set $\mathcal{K}_{t}$ is described by the positive semidefiniteness of an affinely parametrized matrix, it is an instance of a spectrahedron, cf. Chapter ??? of this volume. The following lemma is the truncated analogue of Lemma 4.

Lemma 6. (Generic truncated linear forms) The following assertions are equivalent for $\Lambda \in\left(\mathbb{R}[x]_{t}\right)^{*}$ :

(i) $\operatorname{rank} M_{\lfloor t / 2\rfloor}(\Lambda) \geq \operatorname{rank} M_{\lfloor t / 2\rfloor}\left(\Lambda^{\prime}\right)$ for all $\Lambda^{\prime} \in \mathcal{K}_{t}$.

(ii) $\operatorname{Ker} M_{\lfloor t / 2\rfloor}(\Lambda) \subseteq \operatorname{Ker} M_{\lfloor t / 2\rfloor}\left(\Lambda^{\prime}\right)$ for all $\Lambda^{\prime} \in \mathcal{K}_{t}$.

(iii) The linear form $\Lambda$ lies in the relative interior of the convex set $\mathcal{K}_{t}$. 
Then $\Lambda$ is called a generic element of $\mathcal{K}_{t}$ and the kernel $\mathcal{N}_{t}=\operatorname{Ker} M_{\lfloor t / 2\rfloor}(\Lambda)$ is independent of the particular choice of the generic element $\Lambda \in \mathcal{K}_{t}$.

Theorem 10. We have: $\mathcal{N}_{t} \subseteq \mathcal{N}_{t+1} \subseteq \ldots \subseteq \sqrt[\mathbb{R}]{I}$, with equality $\sqrt[\mathbb{R}]{I}=\left\langle\mathcal{N}_{t}\right\rangle$ for $t$ large enough.

Proof. Let $\Lambda \in \mathcal{K}_{t+1}$ be generic. Its restriction to $\left(\mathbb{R}[x]_{t}\right)^{*}$ lies in $\mathcal{K}_{t}$, implying

$$
\mathcal{N}_{t+1}=\operatorname{Ker} M_{\lfloor(t+1) / 2\rfloor}(\Lambda) \supseteq \operatorname{Ker} M_{\lfloor t / 2\rfloor}(\Lambda) \supseteq \mathcal{N}_{t} .
$$

Now let $\Lambda$ be a generic element of $\mathcal{K}_{t}$ so that $\mathcal{N}_{t}=\operatorname{Ker} M_{\lfloor t / 2\rfloor}(\Lambda)$. The inclusion: $\mathcal{N}_{t} \subseteq \mathcal{I}\left(V_{\mathbb{R}}(I)\right)$ follows using Lemma 6 (ii). Indeed, $\Lambda_{v} \in \mathcal{K}_{t}$ for all $v \in V_{\mathbb{R}}(I)$, which implies $\operatorname{Ker} M_{\lfloor t / 2\rfloor}(\Lambda) \subseteq \operatorname{Ker} M_{\lfloor t / 2\rfloor}\left(\Lambda_{v}\right) \subseteq \mathcal{I}(v)$ and thus $\operatorname{Ker} M_{\lfloor t / 2\rfloor}(\Lambda) \subseteq \bigcap_{v \in V_{\mathbb{R}}(I)} \mathcal{I}(v)=\mathcal{I}\left(V_{\mathbb{R}}(I)\right)=\sqrt[\mathbb{R}]{I}$ (by the Real Nullstellensatz).

We now show equality: $\sqrt[\mathbb{R}]{I}=\left\langle\mathcal{N}_{t}\right\rangle$ for $t$ large enough. For this, let $\left\{g_{1}, \ldots, g_{L}\right\}$ be a basis of the ideal $\sqrt[\mathbb{R}]{I}$; we show that $g_{l} \in \mathcal{N}_{t}$ for all $l$. We have:

$$
g_{l}^{2 k}+\sum_{j} s_{j}^{2}=\sum_{i=1}^{m} u_{i} h_{i} \text { for some } k \in \mathbb{N} \text { and } s_{j}, u_{i} \in \mathbb{R}[x] .
$$

Since $\Lambda \in \mathcal{H}_{t}^{\perp}$, we have $h_{i} \in \mathcal{N}_{t}$ if $t \geq 2 \operatorname{deg}\left(h_{i}\right)$. Using Lemma 5 (ii), this implies that, for $t$ large enough, $\mathcal{N}_{t}$ contains each $u_{i} h_{i}$ and thus $g_{l}^{2 k}+\sum_{j} s_{j}^{2}$. In particular, $\Lambda\left(g_{l}^{2 k}+\sum_{j} s_{j}^{2}\right)=0$. On the other hand, $\Lambda\left(g_{l}^{2 k}\right), \Lambda\left(s_{j}^{2}\right) \geq 0$ (since $M_{\lfloor t / 2\rfloor}(\Lambda) \succeq 0$ ), thus implying $\Lambda\left(g_{l}^{2 k}\right)=0$. An easy induction on $k$ now permits to conclude that $g_{l} \in \mathcal{N}_{t}$.

When $V_{\mathbb{R}}(I)$ is finite, one can guaranty the equality $\sqrt[\mathbb{R}]{I}=\left\langle\mathcal{N}_{t}\right\rangle$ using the rank condition (11). The next results provide all the ingredients of the moment matrix algorithm for real roots, whose description is given in Section 3.3: Theorem 11 will provide a stopping criterion (when $\left|V_{\mathbb{R}}(I)\right|<\infty$ ) and Theorem 12 below will imply its termination, as well as provide a criterion permitting to check the (non-)existence of real roots.

Theorem 11. [17] Let $I=\left\langle h_{1}, \ldots, h_{m}\right\rangle$ be an ideal in $\mathbb{R}[x], D=\max _{i} \operatorname{deg}\left(h_{i}\right)$, and $d=\lceil D / 2\rceil$. Let $\Lambda \in \mathcal{K}_{t}$ be a generic element and assume that at least one of the following two conditions holds:

$$
\begin{aligned}
& \operatorname{rank} M_{s}(\Lambda)=\operatorname{rank} M_{s-1}(\Lambda) \quad \text { for some } D \leq s \leq\lfloor t / 2\rfloor, \\
& \operatorname{rank} M_{s}(\Lambda)=\operatorname{rank} M_{s-d}(\Lambda)
\end{aligned}
$$

Then, $\sqrt[\mathbb{R}]{I}=\left\langle\operatorname{Ker} M_{s}(\Lambda)\right\rangle$, and any basis of the column space of $M_{s-1}(\Lambda)$ is a basis of the quotient space $\mathbb{R}[x] / \sqrt[\mathbb{R}]{I}$.

Proof. The ideal $J:=\left\langle\operatorname{Ker} M_{s}(\Lambda)\right\rangle$ is real radical (by Theorem 9). Moreover,

$$
\operatorname{Ker} M_{s}(\Lambda) \subseteq \operatorname{Ker} M_{\lfloor t / 2\rfloor}(\Lambda) \subseteq \sqrt[\mathbb{R}]{I}
$$


(since $\Lambda$ is generic and using Theorem 10) and thus $J \subseteq \sqrt[\mathbb{R}]{I}$. Remains to show $\sqrt[\mathbb{R}]{I} \subseteq J$. Suppose first that (14) holds. The condition $\Lambda \in \mathcal{H}_{t}^{\perp}$ implies that $h_{i} \in \operatorname{Ker} M_{s}(\Lambda)$ (since $s+\operatorname{deg}\left(h_{i}\right) \leq t / 2+\operatorname{deg}\left(h_{i}\right) \leq t$, as $t \geq 2 D$ ). Thus $I \subseteq J$, implying $\sqrt[\mathbb{R}]{I} \subseteq J$ as $J$ is real radical.

Suppose now that (15) holds. Again from Theorem 9 we know that $V_{\mathbb{C}}\left(\operatorname{Ker} M_{s}(\Lambda)\right)=\left\{v_{1}, \ldots, v_{r}\right\} \subseteq \mathbb{R}^{n}$ and $\Lambda=\sum_{i=1}^{r} \lambda_{i} \Lambda_{v_{i}}$ where $\lambda_{i}>0$. Let $p_{1}, \ldots, p_{r}$ be interpolation polynomials at the $v_{i}$ 's, i.e., such that $p_{j}\left(v_{i}\right)=\delta_{i, j}$. An easy but crucial observation (made in [20]) is that we may assume that each $p_{j}$ has degree at most $s-d$. Indeed, we can replace each interpolation polynomial $p_{j}$ by its normal form modulo $J$ with respect to a basis of $\mathbb{R}[x] / J$. As such a basis can be obtained by picking a column basis of $M_{s-d}(\Lambda)$, its members are monomials of degree at most $s-d$, and the resulting normal forms of the $p_{j}$ 's are again interpolation polynomials at the $v_{i}$ 's but now with degree at most $s-d$. As $\operatorname{deg}\left(p_{j}^{2}\right) \leq 2(s-d) \leq t-2 d \leq t-\operatorname{deg}\left(h_{i}\right)$, we can claim that $\Lambda\left(p_{j}^{2} h_{i}\right)=0$ and in turn $0=\Lambda\left(p_{j}^{2} h_{i}\right)=\sum_{l=1}^{r} \lambda_{l} p_{j}^{2}\left(v_{l}\right) h_{i}\left(v_{l}\right)=\lambda_{j} h_{i}\left(v_{j}\right)$. Since $h_{i}\left(v_{j}\right)=0$ for all $i, j$, we conclude that $\left\{v_{1}, \ldots, v_{r}\right\} \subseteq V_{\mathbb{R}}(I)$, implying the desired inclusion $\sqrt[\mathbb{R}]{I}=\mathcal{I}\left(V_{\mathbb{R}}(I)\right) \subseteq \mathcal{I}\left(v_{1}, \ldots, v_{r}\right)=J$.

Theorem 12. [17] Let $I$ be an ideal in $\mathbb{R}[x]$.

(i) If $V_{\mathbb{R}}(I)=\emptyset$, then $\mathcal{K}_{t}=\emptyset$ for $t$ large enough.

(ii) If $1 \leq\left|V_{\mathbb{R}}(I)\right|<\infty$ then, for $t$ large enough, there exists an integer $s$ for which (15) holds for all $\Lambda \in \mathcal{K}_{t}$.

Proof. Let $\left\{g_{1}, \ldots, g_{L}\right\}$ be a Gröbner basis of $\sqrt[\mathbb{R}]{I}$ with respect to a total degree monomial ordering, and let $\mathcal{B}$ be the corresponding set of standard monomials, forming a basis of $\mathbb{R}[x] / \sqrt[\mathbb{R}]{I}$. The argument used in the proof of Theorem 10 shows the existence of $t_{0} \in \mathbb{N}$ for which $\left\{g_{1}, \ldots, g_{L}\right\} \subseteq \operatorname{Ker} M_{\lfloor t / 2\rfloor}(\Lambda)$ for all $t \geq t_{0}$ and $\Lambda \in \mathcal{K}_{t}$.

(i) If $V_{\mathbb{R}}(I)=\emptyset$, then $\{1\}$ is a basis of $\sqrt[\mathbb{R}]{I}=\mathbb{R}[x]$. Thus $1 \in \operatorname{Ker} M_{\lfloor t / 2\rfloor}(\Lambda)$, implying $\Lambda(1)=0$ if $\Lambda \in \mathcal{K}_{t}$, contradicting $\Lambda(1)=1$ and thus showing $\mathcal{K}_{t}=\emptyset$.

(ii) As $V_{\mathbb{R}}(I)$ is finite, $s:=d+\max _{b \in \mathcal{B}} \operatorname{deg}(b)$ is well defined. Recall that $d=\max _{i}\left\lceil\operatorname{deg}\left(h_{i}\right) / 2\right\rceil$. Choose $t \geq t_{0}$ such that $s<\lfloor t / 2\rfloor$. For $\alpha \in \mathbb{N}_{s}^{n}$, decompose $x^{\alpha}$ as

$$
x^{\alpha}=\sum_{b \in \mathcal{B}} \lambda_{b} b+\sum_{l=1}^{L} u_{l} g_{l} \in \operatorname{Span}_{\mathbb{R}}(\mathcal{B}) \oplus \sqrt[\mathbb{R}]{I},
$$

where $\lambda_{b} \in \mathbb{R}, u_{l} \in \mathbb{R}[x], \operatorname{deg}\left(\sum_{b} \lambda_{b} b\right) \leq s-d$, and $\operatorname{deg}\left(u_{l} g_{l}\right) \leq s<\lfloor t / 2\rfloor$ (as the $g_{l}$ 's form a Gröbner basis for a total degree ordering, we can claim $\left.\operatorname{deg}\left(u_{l} g_{l}\right) \leq s\right)$. As $g_{l} \in \operatorname{Ker} M_{\lfloor t / 2\rfloor}(\Lambda)$ and $\operatorname{deg}\left(u_{l} g_{l}\right)<\lfloor t / 2\rfloor$, we also have that $u_{l} g_{l} \in \operatorname{Ker} M_{\lfloor t / 2\rfloor}(\Lambda)$ (recall Lemma 5 (ii)). Hence, $x^{\alpha}-\sum_{b \in \mathcal{B}} \lambda_{b} b \in$ Ker $M_{\lfloor t / 2\rfloor}(\Lambda)$, which shows that $M_{s}(\Lambda)$ is a flat extension of $M_{s-d}(\Lambda)$.

Example 9 (Example 7 cont.). Consider again the ideal $I=\left(h_{1}, h_{2}, h_{3}\right)$ from Example 7 . Then, $D=5, \operatorname{dim} \mathbb{R}[x] / I=9$, and the variety $V_{\mathbb{C}}(I)$ consists of 
two real points, one of them with multiplicity eight. Table 1 shows the ranks of the moment matrix $M_{s}(\Lambda)$ for generic $\Lambda \in \mathcal{K}_{t}$. The rank condition holds at order $(t, s)=(6,2)$. Then we can extract the two roots $v_{1}=(0,0)$ and $v_{2}=(1,2)$ as well as the (border) basis $\left\{g_{1}, g_{2}, g_{3}\right\}$ of $\sqrt[\mathbb{R}]{I}$ (already discussed in Example 7). This is possible although here $s=2$ is strictly smaller than $d=3$ and $D=5$; indeed, in view of Theorem 5 , we can simply check whether the formal multiplication matrices commute and whether $h_{i}(v)=0$ for all $i=1, \ldots, m$ and $v \in V_{\mathbb{C}}\left(\operatorname{Ker} M_{s}(\Lambda)\right)$.

\begin{tabular}{|c|c|c|c|c|}
\hline$s=$ & 0 & 1 & 2 & 3 \\
\hline$t=5$ & 1 & 3 & 5 & - \\
\hline $\bar{t}=6$ & 1 & 2 & 2 & 4 \\
\hline
\end{tabular}

Table 1. Ranks of $M_{s}(\Lambda)$ for generic $\Lambda \in \mathcal{K}_{t}$ in Example 9.

We conclude with two remarks about Theorem 11, which is the central result of this section. Namely we mention a generalization and an application.

First, observe that one may work with moment matrices $M_{\mathcal{B}}(y)$ indexed by an arbitrary monomial set $\mathcal{B}$, instead of moment matrices $M_{t}(y)$ indexed by all monomials up to a given degree $t$, which leads to possible generalizations of Theorem 11. More precisely, let $\Lambda$ be a generic element in $\mathcal{K}_{t}$. Assume that we can find a monomial set $\mathcal{B}$, connected to 1 , indexing a linearly independent set of columns of the moment matrix $M_{\lfloor t / 2\rfloor}(\Lambda)$, and for which the submatrices $M_{\mathcal{B}}(\Lambda)$ and $M_{\mathcal{B}^{+}}(\Lambda)$ indexed, respectively, by $\mathcal{B}$ and $\mathcal{B}^{+}$, satisfy the rank condition:

$$
\operatorname{rank} M_{\mathcal{B}^{+}}(\Lambda)=\operatorname{rank} M_{\mathcal{B}}(\Lambda) .
$$

Then one can show that the ideal $J=\left\langle\operatorname{Ker} M_{\mathcal{B}^{+}}(\Lambda)\right\rangle$ is real radical, zerodimensional, and contained in $\sqrt[\mathbb{R}]{I}$, and thus $V_{\mathbb{R}}(I) \subseteq V_{\mathbb{C}}(J)$; this result relies on a generalization of the flat extension theorem (Theorem 8 ) proved in $[22$, Thm. 1.4]. Hence, one can compute the variety $V_{\mathbb{C}}(J) \subseteq \mathbb{R}^{n}$, and select from it the desired real variety $V_{\mathbb{R}}(I)$.

Next, as a byproduct of Theorem 11, we see that the rank condition (14) (or (15)) also implies a full description of the convex hull of the variety $V_{\mathbb{R}}(I)$. Indeed, under (14), we can apply Theorem 9 to deduce that, for any $\Lambda \in \mathcal{K}_{t}$, its restriction $\pi_{2 s}(\Lambda)$ can be written as a conic combination of evaluations at points of $V_{\mathbb{R}}(I)$. Combining with Theorem 12, we obtain:

Corollary 1. Assume $1 \leq\left|V_{\mathbb{R}}(I)\right|<\infty$. For some integers $1 \leq s \leq\lfloor t / 2\rfloor$, the set

$$
\left\{\left(\Lambda\left(x^{\alpha}\right)\right)_{\alpha \in \mathbb{N}_{2 s}^{n}} \mid \Lambda \in \mathcal{K}_{t}\right\}
$$

is equal to the convex hull of the set $\left\{[v]_{2 s} \mid v \in V_{\mathbb{R}}(I)\right\}$.

Chapter ??? in this volume considers in detail the problem of characterizing the convex hull of a real variety $V_{\mathbb{R}}(I)$. Although the points of view and emphasis are different in both chapters, there are some connections. Roughly 
speaking, both chapters can be cast within the more general realm of polynomial optimization (see Section 4.1); however, while we work here with truncated sections of the ideal $I$, Chapter ??? deals with linear forms on the full quotient space $\mathbb{R}[x] / I$.

\subsection{The moment matrix algorithm for computing real roots}

We now describe the moment matrix algorithm for computing real roots, summarized in Algorithm 1 below.

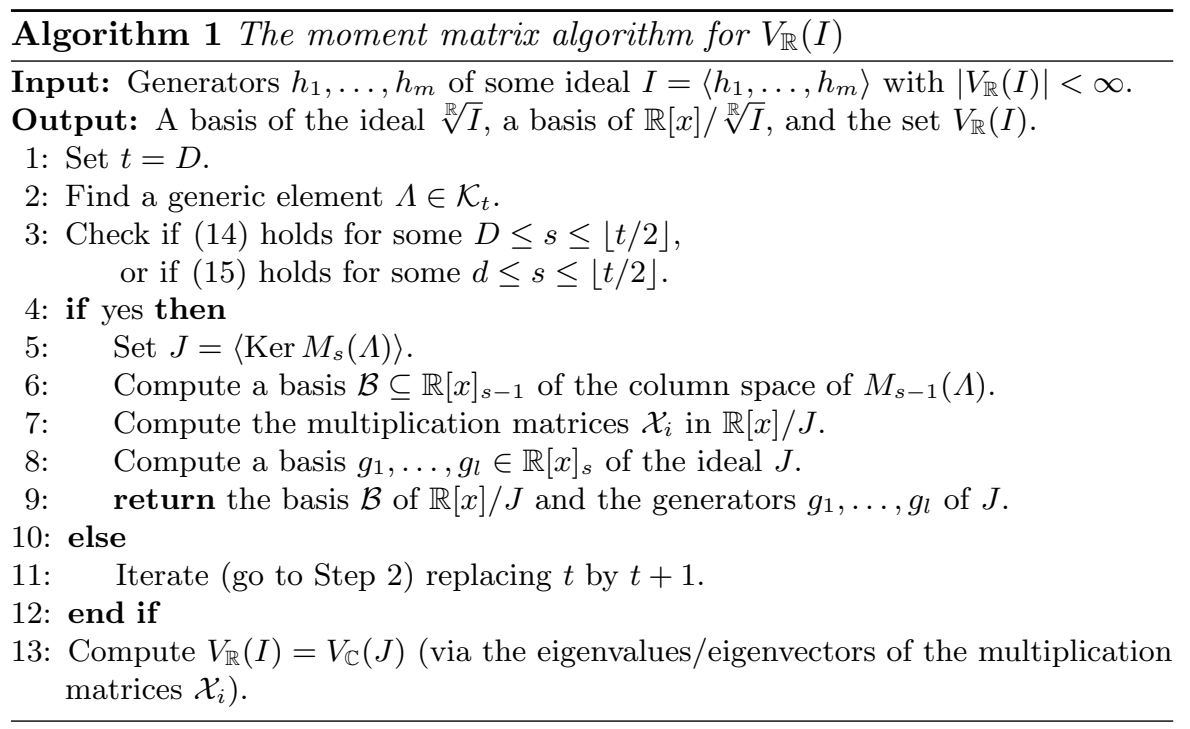

Theorem 11 implies the correctness of the algorithm (i.e., equality $J=\sqrt[\mathbb{R}]{I}$ ) and Theorem 12 shows its termination. Algorithm 1 consists of four main parts, which we now briefly discuss (see $[17,36]$ for details).

(i) Finding a generic element in $\mathcal{K}_{t}$. The set $\mathcal{K}_{t}$ can be represented as the feasible region of a semidefinite program and we have to find a point lying in its relative interior. Such a point can be found by solving several semidefinite programs with an arbitrary SDP solver (cf. [17, Remark 4.15]), or by solving a single semidefinite program with an interior-point algorithm using a self-dual embedding technique (see, e.g., [8], [45]). Indeed consider the semidefinite program:

$$
\begin{aligned}
\min _{\Lambda \in\left(\mathbb{R}[x]_{t}\right)^{*}} 1 \text { such that } & \Lambda(1)=1, M_{\lfloor t / 2\rfloor}(\Lambda) \succeq 0, \\
& \Lambda\left(h_{i} x^{\alpha}\right)=0 \forall i \forall|\alpha| \leq t-\operatorname{deg}\left(h_{i}\right),
\end{aligned}
$$

whose dual reads: 
$\max \lambda$ such that $1-\lambda=s+\sum_{i=1}^{m} u_{i} h_{i}$ where $s, u_{i} \in \mathbb{R}[x]$, $s$ is a sum of squares, $\operatorname{deg}(s), \operatorname{deg}\left(u_{i} h_{i}\right) \leq t$.

The feasible region of (16) is the set $\mathcal{K}_{t}$, as well as its set of optimal solutions, since we minimize a constant objective function over $\mathcal{K}_{t}$. There is no duality gap, as $\lambda=1$ is obviously feasible for (17). Solving the program (16) with an interior-point algorithm using a self-dual embedding technique yields ${ }^{4}$ either a solution $\Lambda$ lying in the relative interior of the optimal face (i.e., a generic element of $\mathcal{K}_{t}$ ), or a certificate that (16) is infeasible thus showing $V_{\mathbb{R}}(I)=\emptyset$.

(ii) Computing the ranks of submatrices of $M_{t}(\Lambda)$. In order to check whether one of the conditions (14) or (15) holds we need to compute the ranks of matrices consisting of numerical values. This computationally challenging task may be done by detecting zero singular values and/or a large decay between two subsequent values.

(iii) Computing a basis $\mathcal{B}$ for the column space of $M_{s-1}(\Lambda)$. The set of monomials $\mathcal{B}$ indexing a maximum nonsingular principle submatrix of $M_{s}(\Lambda)$ directly reveals a basis of the quotient space $\mathbb{R}[x] / J$ (by Theorem 9 ). The choice of this basis may influence the numerical stability of the extracted set of solutions and the properties of the border basis of $J$ as well. The options range from a monomial basis obtained using a greedy algorithm or more sophisticated polynomial bases (see [36]).

(iv) Computing a basis of $J$ and the formal multiplication matrices. Say $\mathcal{B}$ is the monomial basis (connected to 1 ) of the column space of $M_{s-1}(\Lambda)$ constructed at the previous step (iii). Under the rank condition (14) or (15), for any $b \in \mathcal{B}$, the monomial $x_{i} b$ can be written as $x_{i} b=r_{i, b}+q$, where $r_{i, b} \in$ $\operatorname{Span}_{\mathbb{R}}(\mathcal{B})$ and $q \in \operatorname{Ker} M_{s}(\Lambda)$. These polynomials directly give a (border) basis of $J$, consisting of the polynomials $\left\{x_{i} b-r_{i, b} \mid i \leq n, b \in \mathcal{B}\right\}$ (recall Theorem 5) and thus permit the construction of multiplication matrices and the computation of $V_{\mathbb{C}}(J)\left(=V_{\mathbb{R}}(I)\right)$.

Existing implementations and performance. The basic algorithm discussed above has been implemented in Matlab using Yalmip (see [25]) as part of a new toolbox Bermeja for computations in Convex Algebraic Geometry (see [37]). In its current form, the implemented algorithm merely provides a proof of concept and only solves real root finding problems with a rather limited number of variables $(\leq 10)$ and of moderate degree $(\leq 6)$. This is mainly due to the fact that sparsity in the support of the polynomials is not utilized. This leads to large moment matrices, easily touching on the limitations of current SDP solver. We refer to $[17,19]$ for a more detailed discussion and some numerical results. In an ongoing project a more efficient, Buchbergerstyle, version of this real root finding method will be implemented based on the more general version of the flat extension theorem, which was described at

\footnotetext{
${ }^{4}$ This follows under certain technical conditions on the semidefinite program, which are satisfied for (16); see [17] for details.
} 
the end of Section 3.2. A flavor of how existing complex root finding methods may be tailored for real root finding is discussed in the next section.

\subsection{Real vs. complex root finding}

As we saw in the previous section, the moment matrix approach for real roots relies on finding a suitable (generic) linear form $\Lambda$ in the convex set $\mathcal{K}_{t}$ (from (13)). Let us stress again that the positivity condition on $\Lambda$ is the essential ingredient that permits to focus solely on the real roots among the complex ones. This is best illustrated by observing (following [18]) that, if we delete the positivity condition in the moment matrix algorithm (Algorithm 1), then the same algorithm permits to compute all complex roots (assuming their number is finite). In other words, consider the following analogue of the set $\mathcal{K}_{t}$ :

$$
\mathcal{K}_{t}^{\mathbb{C}}=\left\{\Lambda \in\left(\mathbb{R}[x]_{t}\right)^{*} \mid \Lambda(1)=1 \text { and } \Lambda(f)=0 \forall f \in \mathcal{H}_{t}\right\},
$$

where $\mathcal{H}_{t}$ is as in (12). Call an element $\Lambda \in \mathcal{K}_{t}^{\mathbb{C}}$ generic $^{5}$ if $\operatorname{rank} M_{s}(\Lambda)$ is maximum for all $s \leq\lfloor t / 2\rfloor$. Then the moment matrix algorithm for complex roots is analogous to Algorithm 1, but with the following small twist: Instead of computing a generic element in the convex set $\mathcal{K}_{t}$, we have to compute a generic (aka random) element in the affine space $\mathcal{K}_{t}^{\mathbb{C}}$, thus replacing the semidefinite feasibility problem by a linear algebra computation. We refer to [18] for details on correctness and termination of this algorithm.

Alternatively one can describe the above situation as follows: the complex analogue of Algorithm 1 is an algorithm for complex roots, which can be turned into an algorithm for real roots simply by adding the positivity condition on $\Lambda$. This suggests that the same recipe could be applied to other algorithms for complex roots. This is indeed the case, for instance, for the prolongation-projection algorithm of [35] which, as shown in [19], can be turned into an algorithm for real roots by adding a positivity condition. The algorithm of [35] works with the space $\mathcal{K}_{t}^{\mathbb{C}}$ but uses a different stopping criterion instead of the rank condition (14). Namely one should check whether, for some $D \leq s \leq t$, the three affine spaces $\pi_{s}\left(\mathcal{K}_{t}^{\mathbb{C}}\right), \pi_{s-1}\left(\mathcal{K}_{t}^{\mathbb{C}}\right)$, and $\pi_{s}\left(\mathcal{K}_{t+1}^{\mathbb{C}}\right)$ have the same dimensions (where $\pi_{s}(\Lambda)$ denotes the restriction of $\Lambda \in\left(\mathbb{R}[x]_{t}\right)^{*}$ to $\left.\left(\mathbb{R}[x]_{s}\right)^{*}\right)$; if so, one can compute a basis of $\mathbb{R}[x] / I$ and extract $V_{\mathbb{C}}(I)$. Roughly speaking, to turn this into an algorithm for real roots, one adds positivity and considers the convex set $\mathcal{K}_{t}$ instead of $\mathcal{K}_{t}^{\mathbb{C}}$; again one needs to check that three suitably defined spaces have the same dimensions; if so, then one can extract $V_{\mathbb{R}}(I)$. We refer to [19] for details, also about the links between the rank condition and the above alternative stopping criterion.

\footnotetext{
${ }^{5}$ When $\Lambda$ is positive, the maximality condition on the rank of $M_{\lfloor t / 2\rfloor}(\Lambda)$ implies that the rank of $M_{s}(\Lambda)$ is maximum for all $s \leq\lfloor t / 2\rfloor$. This is not true for $\Lambda$ non-positive.
} 
Example 10. We apply the real vs. complex moment matrix algorithms to the ideal $I=\left\langle h_{1}, h_{2}, h_{3}\right\rangle$ (taken from [5, Ex. 4, p.57]), where

$$
h_{1}=x_{1}^{2}-2 x_{1} x_{3}+5, \quad h_{2}=x_{1} x_{2}^{2}+x_{2} x_{3}+1, \quad h_{3}=3 x_{2}^{2}-8 x_{1} x_{3},
$$

with $D=3,\left|V_{\mathbb{C}}(I)\right|=8$ and $\left|V_{\mathbb{R}}(I)\right|=2$. Table 2 shows the ranks of the generic moment matrices when applying the real vs. complex versions of the moment matrix algorithm. We see that the algorithm terminates earlier in the real case, namely at order $t=6$, compared to order $t=9$ in the complex case. If we replace each polynomial $h_{i}$ by $h_{i} \cdot\left(1+\sum_{i} x_{1}^{2}\right)$, we obtain an example with a positive dimensional complex variety, while the real variety is unchanged. The real root finding algorithm still terminates (now at order $t=7$ ) and allows the extraction of the two real roots.

(a) Generic $\Lambda \in \mathcal{K}_{t}$

(b) Generic $\Lambda \in \mathcal{H}_{t}^{\perp}$

\begin{tabular}{c|cccc}
$s=$ & 0 & 1 & 2 & 3 \\
\hline \hline$t=2$ & 1 & 4 & - & - \\
\hline$t=3$ & 1 & 4 & - & - \\
\hline$t=4$ & 1 & 4 & 8 & - \\
\hline$t=5$ & 1 & 2 & 8 & - \\
\hline$t=6$ & 1 & $\mathbf{2}$ & $\mathbf{2}$ & 10 \\
\hline
\end{tabular}

\begin{tabular}{c|ccccc}
\multicolumn{1}{c}{$s=$} & 0 & 1 & 2 & 3 & 4 \\
\hline \hline$t=2$ & 1 & 4 & - & - & - \\
\hline$t=3$ & 1 & 4 & - & - & - \\
\hline$t=4$ & 1 & 4 & 8 & - & - \\
\hline$t=5$ & 1 & 4 & 8 & - & - \\
\hline$t=6$ & 1 & 4 & 8 & 11 & - \\
\hline$t=7$ & 1 & 4 & 8 & 10 & - \\
\hline$t=8$ & 1 & 4 & 8 & 9 & 10 \\
\hline$t=9$ & 1 & 4 & $\mathbf{8}$ & $\mathbf{8}$ & 10 \\
\hline
\end{tabular}

Table 2. Ranks of $M_{s}(\Lambda)$ in Example 10 .

\section{Further directions and connections}

The moment approach for real solving polynomial equations can be extended and applied in various directions. We briefly mentioned at the end of Section 3.2 the link to the approach of Chapter ??? for approximating the convex hull of a real variety. We now touch a few selected extensions: polynomial optimization, emptyness certificates for real varieties, the positive dimensional case, and quotient ideals.

\subsection{Optimization and polynomial inequalities}

The research field of polynomial optimization, which roots, in particular, in work of Lasserre [15], Parrilo [34], Shor [39], has recently undergone a spectacular development. We refer e.g. to the monograph [16] or the survey [21] for overview and further references. The moment approach was originally proposed in [15] for solving general nonlinear optimization problems of the form 


$$
\begin{aligned}
f^{*}=\min _{x} f(x) \text { such that } & h_{1}(x)=0, \ldots, h_{m}(x)=0, \\
g_{1}(x) & \geq 0, \ldots, g_{p}(x) \geq 0,
\end{aligned}
$$

where $f, h_{i}, g_{j} \in \mathbb{R}[x]$. Let $I=\left\langle h_{1}, \ldots, h_{m}\right\rangle$ be the ideal generated by the $h_{i}$ 's, and set

$$
S=\left\{x \in \mathbb{R}^{n} \mid g_{1}(x) \geq 0, \ldots, g_{p}(x) \geq 0\right\},
$$

so that (19) asks to minimize $f$ over the semi-algebraic set $V_{\mathbb{R}}(I) \cap S$. The basic observation in [15] is that the problem (19) can be reformulated as

$$
\min _{\mu} \Lambda_{\mu}(f) \text { such that } \mu \text { is a probability measure on } V_{\mathbb{R}}(I) \cap S \text {, }
$$

where $\Lambda_{\mu}$ is as in (9). Such a linear form satisfies: $\Lambda(h)=0$ for all $h \in I$, as well as the positivity condition: $\Lambda\left(g_{j} f^{2}\right) \geq 0$ for all $f \in \mathbb{R}[x]$ and $j=1, \ldots, p$. The latter conditions can be reformulated as requiring that the localizing moment matrices $M_{\left\lfloor\frac{t-\operatorname{deg}\left(g_{j}\right)}{2}\right\rfloor}\left(g_{j} \Lambda\right)$ be positive semidefinite. Here, for $g \in \mathbb{R}[x], g \Lambda$ is the new linear form defined by $g \Lambda(p)=\Lambda(p g)$ for all $p \in \mathbb{R}[x]$.

The semidefinite program (16) can be modified in the following way to yield a relaxation of (19):

$$
\begin{aligned}
f_{t}^{*}=\min _{\Lambda \in\left(\mathbb{R}[x]_{t}\right)^{*}} \Lambda(f) \text { such that } & \Lambda(1)=1, \Lambda(h)=0 \forall h \in \mathcal{H}_{t}, \\
& M_{\left\lfloor\frac{t-\operatorname{deg}\left(g_{j}\right)}{2}\right\rfloor}\left(g_{j} \Lambda\right) \succeq 0(j=0,1, \ldots, p)
\end{aligned}
$$

(setting $g_{0}=1$ ). The dual semidefinite program reads:

$$
\max \lambda \text { such that } f-\lambda=\sum_{j=0}^{p} \sigma_{j} g_{j}+\sum_{i=1}^{m} u_{i} h_{i}
$$

where $u_{i} \in \mathbb{R}[x], \sigma_{j}$ are sums of squares of polynomials with $\operatorname{deg}\left(u_{i} h_{i}\right)$, $\operatorname{deg}\left(\sigma_{j} g_{j}\right) \leq t$. Then, $f_{t}^{*} \leq f^{*}$ for all $t$. Moreover, asymptotic convergence of (21) and (22) to the minimum $f^{*}$ of (19) can be shown when the feasible region of (19) is compact and satisfies some additional technical condition (see [15]). We now group some results showing finite convergence under certain rank condition, which can be seen as extensions of Theorems 11 and 12 .

Theorem 13. [12, 17, 21] Let $D:=\max _{i, j}\left(\operatorname{deg}\left(h_{i}\right), \operatorname{deg}\left(g_{j}\right)\right), d:=\lceil D / 2\rceil$, $t \geq \max (\operatorname{deg}(f), D)$, and let $\Lambda$ be a generic optimal solution to (21) (i.e., for which rank $M_{\lfloor t / 2\rfloor}(\Lambda)$ is maximum), provided it exists.

(i) If the rank condition (15) holds with $2 s \geq \operatorname{deg}(f)$, then $f_{t}^{*}=f^{*}$ and $V_{\mathbb{C}}\left(\operatorname{Ker} M_{s}(\Lambda)\right)$ is equal to the set of global minimizers of the program (19).

(ii) If $V_{\mathbb{R}}(I)$ is nonempty and finite, then (15) holds with $2 s \geq \operatorname{deg}(f)$.

(iii) If $V_{\mathbb{R}}(I)=\emptyset$, then the program (21) is infeasible for $t$ large enough. 
In other words, under the rank condition (15), one can compute all global minimizers of the program (19), since, as before, one can compute a basis of the space $\mathbb{R}[x] /\left\langle\operatorname{Ker} M_{s}(\Lambda)\right\rangle$ from the moment matrix and thus apply the eigenvalue method. Moreover, when the equations $h_{i}=0$ have finitely many real roots, the rank condition is guaranteed to hold after finitely many steps.

By choosing the constant objective function $f=1$ in (19), we can also compute the $S$-radical ideal:

$$
\sqrt[S]{I}:=\mathcal{I}\left(V_{\mathbb{R}}(I) \cap S\right) .
$$

When $\left|V_{\mathbb{R}}(I)\right|$ is nonempty and finite, one can show that

$$
\mathcal{I}\left(V_{\mathbb{R}}(I) \cap S\right)=\left\langle\operatorname{Ker} M_{s}(\Lambda)\right\rangle
$$

for a generic optimal solution $\Lambda$ of (21) and $s, t$ large enough. An analogous result holds under the weaker assumption that $\left|V_{\mathbb{R}}(I) \cap S\right|$ is nonempty and finite. In this case $\Lambda$ needs to be a generic feasible solution of the modified semidefinite program obtained by adding to (21) the positivity conditions:

$$
M_{\left\lfloor\frac{t-\operatorname{deg}(g)}{2}\right\rfloor}(g \Lambda) \succeq 0 \text { for } g=g_{1}^{e_{1}} \cdots g_{p}^{e_{p}} \forall e \in\{0,1\}^{p} .
$$

The key ingredient in the proof is to use the Positivstellensatz to characterize the polynomials in $\mathcal{I}\left(V_{\mathbb{R}}(I) \cap S\right)$ (see [42]) instead of the Real Nullstellensatz (used in Theorem 10 to characterize the polynomials in $\mathcal{I}\left(V_{\mathbb{R}}(I)\right)$ ).

Let us illustrate on an example how to 'zoom in' on selected roots, by incorporating semi-algebraic constraints or suitably selecting the cost function.

Example 11. Consider the following system, known as Katsura 5 (see [13]):

$$
\begin{aligned}
& h_{1}=2 x_{6}^{2}+2 x_{5}^{2}+2 x_{4}^{2}+2 x_{3}^{2}+2 x_{2}^{2}+x_{1}^{2}-x_{1}, \\
& h_{2}=x_{6} x_{5}+x_{5} x_{4}+2 x_{4} x_{3}+2 x_{3} x_{2}+2 x_{2} x_{1}-x_{2}, \\
& h_{3}=2 x_{6} x_{4}+2 x_{5} x_{3}+2 x_{4} x_{2}+x_{2}^{2}+2 x_{3} x_{1}-x_{3}, \\
& h_{4}=2 x_{6} x_{3}+2 x_{5} x_{2}+2 x_{3} x_{2}+2 x_{4} x_{1}-x_{4}, \\
& h_{5}=x_{3}^{2}+2 x_{6} x_{1}+2 x_{5} x_{1}+2 x_{4} x_{1}-x_{5}, \\
& h_{6}=2 x_{6}+2 x_{5}+2 x_{4}+2 x_{3}+2 x_{2}+x_{1}-1,
\end{aligned}
$$

with $D=2,\left|V_{\mathbb{C}}(I)\right|=32$, and $\left|V_{\mathbb{R}}(I)\right|=12$. Table $3($ a) shows the ranks of the generic moment matrices for the moment matrix algorithm to compute $V_{\mathbb{R}}(I)$. At order $(t, s)=(6,3)$, the algorithm finds all twelve real roots.

Next we apply the moment matrix algorithm to compute the real roots in $S=\left\{x \in \mathbb{R}^{6} \mid g(x)=x_{1}-0.5 \geq 0\right\} ;$ the ranks are shown in Table 3(b) and all five elements of $V_{\mathbb{R}}(I) \cap S$ can be computed at order $(t, s)=(4,2)$.

If we are interested e.g. only in the roots in $V_{\mathbb{R}}(I) \cap S$ with the smallest $x_{2}$-coordinate then we minimize the polynomial $x_{2}$ (instead of the constant one polynomial). The moment matrix algorithm now terminates at or$\operatorname{der}(t, s)=(2,1)$ and finds the unique element of $V_{\mathbb{R}}(I) \cap S$ with the smallest $x_{2}$-coordinate. 
(a) Generic $\Lambda \in \mathcal{K}_{t}$

(b) Generic $\Lambda \in \mathcal{K}_{t}$ with

$M_{\left\lfloor\frac{t-1}{2}\right\rfloor}(g \Lambda) \succeq 0$

Table 3. Ranks of $M_{s}(\Lambda)$ in Example 11.

\subsection{Exact certificates of emptiness}

If the moment method is applied to an empty real variety $V_{\mathbb{R}}(I)$ (or subset $\left.V_{\mathbb{R}}(I) \cap S\right)$, then the underlying semidefinite optimization problem is infeasible for $t$ large enough, which thus can be thought of as a numerical certificate of emptiness (see Theorems 12,13). If we solve the semidefinite program (16) with a primal-dual interior point solver and infeasibility is detected, an improving ray is returned, i.e., a solution to the dual problem (17) of the form:

$$
1-\lambda^{*}=\sigma+\sum_{i=1}^{m} u_{i} h_{i} \text { where } \sigma, u_{i} \in \mathbb{R}[x] \text { and } \sigma \text { is a sum of squares, }
$$

with $\lambda^{*}>1$. By scaling both sides with an arbitrary positive number, one can generate a feasible solution of the dual problem (17) with an arbitrary high cost function value, thus certifying infeasibility of the primal problem.

On the other hand, by the Real Nullstellensatz, we know that an algebraic certificate for emptyness of $V_{\mathbb{R}}(I)$ is that $1 \in \sqrt[\mathbb{R}]{I}$, i.e.,

$$
1+\sigma=\sum_{i=1}^{m} u_{i} h_{i} \text { for some } \sigma, u_{i} \in \mathbb{R}[x] \text { where } \sigma \text { is a sum of squares. }
$$

In principle, such a certificate can be directly derived from an improving ray such as (23). The difficulty, however, arise from numerical imprecisions and the certificate computed using semidefinite programming does not hold exactly when all computations are done in floating point arithmetics. We may thus only derive polynomials $u_{i}, \sigma$ satisfying

$$
1+\sigma+\epsilon=\sum_{i=1}^{m} u_{i} h_{i}
$$

where $\epsilon \in \mathbb{R}[x]_{t}$ represents the cumulated error term. However, as shown in [36, Prop. 7.38], this approximate certificate can still be used to produce an exact certificate for the nonexistence of roots in some ball $B_{\delta}$ of radius $\delta$ around the origin. Namely, if $|\epsilon(0)| \ll 1$, then one can compute an explicit 
$\delta$ for which one can prove that $V_{\mathbb{R}}(I) \cap B_{\delta}=\emptyset$. This is illustrated on the following example.

Example 12. Consider the ideal $I=\left\langle h_{1}, h_{2}, h_{3}\right\rangle$ generated by

$$
h_{1}=x_{1}^{4}+x_{2}^{4}+x_{3}^{4}-4, h_{2}=x_{1}^{5}+x_{2}^{5}+x_{3}^{5}-5, h_{3}=x_{1}^{6}+x_{2}^{6}+x_{3}^{6}-6
$$

with $D=6,\left|V_{\mathbb{C}}(I)\right|=120$, and $V_{\mathbb{R}}(I)=\emptyset$. At order $t=6$ already, the primal (moment) problem is infeasible, the solver returns an improving direction for the dual (SOS) problem, and we obtain a numerical certificate of the form (25). The error polynomial $\epsilon \in \mathbb{R}[x]$ is a dense polynomial of degree 6 , its coefficients are smaller than 4.1e-11, with constant term $\epsilon(0)<8.53 \mathrm{e}-14$. Using the conservative estimate of $[36, \S 7.8 .2]$ one can rigorously certify the emptiness of the set $V_{\mathbb{R}}(I) \cap B_{\delta}$ for $\delta=38.8$. In other words, even if we only solved the problem numerically with a rather low accuracy, we still obtain a proof that the ideal $I$ does not have any real root $v \in V_{\mathbb{R}}(I)$ with $\|v\|_{2}<38.8$. By increasing the accuracy of the SDP solver the radius $\delta$ of the ball can be further increased. This example illustrates that it is sometimes possible to draw exact conclusions from numerical computations.

\subsection{Positive dimensional ideals and quotient ideals}

Dealing with positive dimensional varieties is a challenging open problem, already for complex varieties (see e.g. the discussion in [24]). The algorithm presented so far for computing the real variety $V_{\mathbb{R}}(I)$ and the real radical ideal $\sqrt[\mathbb{R}]{I}$ works under the assumption that $V_{\mathbb{R}}(I)$ is finite. Indeed, the rank condition (14) (or (15)) implies that $\operatorname{dim} \mathbb{R}[x] / \sqrt[\mathbb{R}]{I}=\operatorname{rank} M_{s-1}(\Lambda)$ is finite (by Theorem 11). Nevertheless, the moment method can in principle be applied to find a basis of $\sqrt[\mathbb{R}]{I}$ also in the positive dimensional case. Indeed Theorem 10 shows that, for $t$ large enough, the kernel of $M_{\lfloor t / 2\rfloor}(\Lambda)$ (for generic $\Lambda \in \mathcal{K}_{t}$ ) generates the real radical ideal $\sqrt[\mathbb{R}]{I}$. The difficulty however is that it is not clear how to recognize whether equality $\sqrt[\mathbb{R}]{I}=\left\langle\operatorname{Ker} M_{\lfloor t / 2\rfloor}(\Lambda)\right\rangle$ holds in the positive dimensional case. These questions relate in particular to the study of the Hilbert function of $\sqrt[\mathbb{R}]{I}$ (see [36]). An interesting research direction is whether the moment matrix approach can be applied to compute some form of "parametric representation" of the real variety. On some instances it is indeed possible to compute parametric multiplication matrices (see [36] for details).

Another interesting object is the quotient (or colon) ideal

$$
I: g=\{p \in \mathbb{R}[x] \mid p g \in I\}
$$

for an ideal $I$ and $g \in \mathbb{R}[x]$. The moment approach can be easily adapted to find a semidefinite characterization of the ideal $\sqrt[\mathbb{R}]{I}: g=\mathcal{I}\left(V_{\mathbb{R}}(I) \backslash V_{\mathbb{R}}(g)\right)$. Indeed, for generic $\Lambda$, the kernel of the localizing moment matrix of $g \Lambda$ carries all information about this ideal. 
Proposition 1. Let $g \in \mathbb{R}[x]_{k}, \rho:=1+\lceil k / 2\rceil$ and $D=\max _{i} \operatorname{deg}\left(h_{i}\right)$. Let $\Lambda$ be a generic element in $\mathcal{K}_{t+k}$.

(i) $\left\langle\operatorname{Ker} M_{\lfloor t / 2\rfloor}(g \Lambda)\right\rangle \subseteq \sqrt[\mathbb{R}]{I}: g$, with equality for $t$ large enough.

(ii) If the rank condition: $\operatorname{rank} M_{s}(\Lambda)=\operatorname{rank} M_{s-\rho}(\Lambda)$ holds for some $s$ with $\max (D, \rho) \leq s \leq\lfloor t / 2\rfloor$, then $\sqrt[\mathbb{R}]{I}: g=\left\langle\operatorname{Ker} M_{s-\rho+1}(g \Lambda)\right\rangle$.

(iii) If $V_{\mathbb{R}}(I)$ is nonempty finite, then the rank condition in (ii) holds at some order $(t, s)$.

Proof. Note that $p \in \operatorname{Ker} M_{\lfloor t / 2\rfloor}(g \Lambda)$ if and only if $p g \in \operatorname{Ker} M_{\lfloor t / 2\rfloor}(\Lambda)$ when $\operatorname{deg}(p) \leq\lfloor t / 2\rfloor-k$.

(i) As $\Lambda$ is generic, Ker $M_{\lfloor t / 2\rfloor}(\Lambda) \subseteq \sqrt[\mathbb{R}]{I}$, implying $\left\langle\operatorname{Ker} M_{\lfloor t / 2\rfloor}(g \Lambda)\right\rangle \subseteq \sqrt[\mathbb{R}]{I}: g$. The proof of equality for $t$ large enough is similar to the proof of Theorem 10 : Pick a basis $\left\{g_{1}, \ldots, g_{L}\right\}$ of the ideal $\sqrt[\mathbb{R}]{I}: g$, so that each $g_{l} g$ belongs to $\sqrt[\mathbb{R}]{I}$; apply the Real Nullstellensatz to $g_{l} g$ to conclude that, for $t$ large enough, $g_{l} g \in \operatorname{Ker} M_{\lfloor t / 2\rfloor}(\Lambda)$ and thus $g_{l} \in \operatorname{Ker} M_{\lfloor t / 2\rfloor}(g \Lambda)$.

(ii) Assume now $\operatorname{rank} M_{s}(\Lambda)=\operatorname{rank} M_{s-\rho}(\Lambda)$ for $D, \rho \leq s \leq\lfloor t / 2\rfloor$. Then there exists $\tilde{\Lambda} \in \mathbb{R}[x]^{*}$ for which $M(\tilde{\Lambda})$ is a flat extension of $M_{s}(\tilde{\Lambda})$ and $\sqrt[\mathbb{R}]{I}=\operatorname{Ker} M(\tilde{\Lambda})=\left\langle\operatorname{Ker} M_{s-\rho+1}(\Lambda)\right\rangle$ (use Theorems 9 and 11). Therefore, $\sqrt[\mathbb{R}]{I}: g=\operatorname{Ker} M(\tilde{\Lambda}): g=\operatorname{Ker} M(g \tilde{\Lambda})$. One can verify that $M(g \tilde{\Lambda})$ is a flat extension of $M_{s-\rho}(g \tilde{\Lambda})$, which implies that $\operatorname{Ker} M(g \tilde{\Lambda})=\left\langle\operatorname{Ker} M_{s-\rho+1}(g \tilde{\Lambda})\right\rangle$ (using Theorem 9) is thus equal to $\left\langle\operatorname{Ker} M_{s-\rho+1}(g \Lambda)\right\rangle$ (since $\tilde{\Lambda}$ and $\Lambda$ coincide on $\mathbb{R}[x]_{2 s}$ ).

(iii) follows from an easy modification of the proof of Theorem 12.

We conclude this chapter with a small example on quotient ideals.

Example 13. Consider $I=\left\langle x_{2}-x_{1}^{2}, x_{2}^{2}-x_{2}\right\rangle$, with roots $(0,0)$ (double) and $( \pm 1,1)$, and $\sqrt[\mathbb{R}]{I}=\left\langle x_{2}-x_{1}^{2}, x_{2}^{2}-x_{2}, x_{1} x_{2}-x_{1}\right\rangle$. The quotient ideal computation with $g=x_{1}$ terminates at order $(t, s)=(6,3)$ and we obtain that $\sqrt[\mathbb{R}]{I}: g$ is generated by $x_{2}-x_{1}^{2}, x_{2}-1$, with variety $V_{\mathbb{R}}(I) \backslash V_{\mathbb{R}}(g)=\{(-1,1),(1,1)\}$. The corresponding ranks of $M_{s}(\Lambda)$ and $M_{s}(g \Lambda)$ are shown in Table 4.
(a) $\operatorname{rank} M_{s}(\Lambda)$
(b) $\operatorname{rank} M_{s}(g \Lambda)$

\begin{tabular}{c|cccc}
\multicolumn{1}{c}{$s=$} & 0 & 1 & 2 & 3 \\
\hline \hline$t=4$ & 1 & 3 & 3 & - \\
\hline$t=5$ & 1 & 3 & 3 & - \\
\hline$t=6$ & 1 & $\mathbf{3}$ & 3 & $\mathbf{3}$ \\
\hline
\end{tabular}

\begin{tabular}{c||cccc}
$s=$ & 0 & 1 & 2 & 3 \\
\hline \hline$\overline{t=4}$ & 1 & 2 & - & - \\
\hline$t=5$ & 1 & 2 & - & - \\
\hline$t=6$ & 1 & $\mathbf{2}$ & $\mathbf{2}$ & - \\
\hline
\end{tabular}

Table 4. Rank sequences for generic $\Lambda \in \mathcal{K}_{t}$ in Example 13. 


\section{References}

1. N.I. Akhiezer, The Classical Moment Problem, Hafner, 1965.

2. S. Basu, R. Pollack, and M.F. Roy, Algorithms in Real Algebraic Geometry, Springer, 2003.

3. D. Bates and F. Sottile, Khovanskii-Rolle continuation for real solutions, arXiv:0908.4579, 2009.

4. J. Bochnak, M. Coste, and M.-F. Roy, Real Algebraic Geometry, Springer, 1998.

5. D.A. Cox, J.B. Little, and D.B. O'Shea, Using Algebraic Geometry, Springer, 1998.

6. __ Ideals, Varieties, and Algorithms : An Introduction to Computational Algebraic Geometry and Commutative Algebra, Springer, 2005.

7. R.E. Curto and L. Fialkow, Solution of the truncated complex moment problem for flat data, Memoirs of the American Mathematical Society 119 (1996), no. $568,1-62$.

8. E. de Klerk, Aspects of Semidefinite Programming - Interior Point Algorithms and Selected Applications, Kluwer, 2002.

9. A. Dickenstein and I. Z. Emiris (eds.), Solving Polynomial Equations: Foundations, Algorithms, and Applications, Algorithms and Computation in Mathematics, vol. 14, Springer, 2005.

10. M. Elkadi and B. Mourrain, Introduction à la Résolution des Systèmes d'Equations Polynomiaux, Mathématiques et Applications, vol. 59, Springer, 2007.

11. E.K. Haviland, On the momentum problem for distribution functions in more than one dimension. II, American Journal of Mathematics 58 (1936), no. 1, $164-168$.

12. D. Henrion and J.B. Lasserre, Detecting global optimality and extracting solutions in GloptiPoly, In Positive Polynomials in Control, Lecture Notes in Control and Information Sciences, pp. 293-310, Springer, 2005.

13. http://www.mat.univie.ac.at/ neum/glopt/coconut/Benchmark/ Library3/katsura5.mod

14. A. Kehrein and M. Kreuzer, Characterizations of border bases, Journal of Pure and Applied Algebra 196 (2005), 251-270.

15. J.B. Lasserre, Global optimization with polynomials and the problem of moments, SIAM Journal on Optimization 11 (2001), 796-817.

16. __ Moments, Positive Polynomials, and their Applications, Imperial College Press, 2009.

17. J.B. Lasserre, M. Laurent, and P. Rostalski, Semidefinite characterization and computation of real radical ideals, Foundations of Computational Mathematics 8 (2008), no. 5, 607-647.

18. _ A unified approach for real and complex zeros of zero-dimensional ideals., In Emerging Applications of Algebraic Geometry (M. Putinar and S. Sullivant, eds.), vol. 149, Springer, 2009, pp. 125-156.

19. _ A prolongation-projection algorithm for computing the finite real variety of an ideal, Theoretical Computer Science 410 (2009), no. 27-29, 2685-2700.

20. M. Laurent, Revisiting two theorems of Curto and Fialkow, Proceedings of the American Mathematical Society 133 (2005), no. 10, 2965-2976.

21. Sums of squares, moment matrices and optimization over polynomials, In Emerging Applications of Algebraic Geometry (M. Putinar and S. Sullivant, eds.), Springer, 2009, pp. 157-270. 
22. M. Laurent and B. Mourrain, A generalized flat extension theorem for moment matrices, Archiv der Mathematik 93 (2009), no. 1, 87-98.

23. D. Lazard, Resolution des systemes d'equations algebriques, Theoretical Computer Science 15 (1981), 77-110.

24. - Thirty years of polynomial system solving, and now? Journal of Symbolic Computation 44 (2009), 222-231.

25. J. Löfberg, YALMIP: A Toolbox for Modeling and Optimization in MATLAB, Computer Aided Control Systems Design Conference (Taipei, Taiwan), 2004, Available from http://users.isy.liu.se/johanl/yalmip/.

26. H.M. Möller, An inverse problem for cubature formulae, Computational Technologies 9 (2004), 13-20.

27. T. Mora, Solving Polynomial Equation Systems I: The Kronecker-Duval Philosophy, Encyclopedia of Mathematics and its Applications, no. 88, Cambridge University Press, 2003.

28. _ Solving Polynomial Equation Systems II: Macaulay's Paradigm and Gröbner Technology, Encyclopedia of Mathematics and its Applications (v. 2), no. 88, Cambridge University Press, 2005.

29. B. Mourrain, A new criterion for normal form algorithms, AAECC, Lecture Notes in Computer Science, pp. 430-443, 1999.

30. _ Pythagore's dilemma, symbolic - numeric computation and the border basis method, In Symbolic-numeric computation, Trends in Mathematics, pp. 223-243, Birkhäuser, 2007.

31. B. Mourrain and J. P. Pavone, Subdivision methods for solving polynomial equations, Journal of Symbolic Computation 44 (2009), no. 3, 292-306.

32. B. Mourrain and P. Trebuchet, Generalized normal forms and polynomials system solving, In Proc. Intern. Symp. on Symbolic and Algebraic Computation (M. Kauers, ed.), pp. 253-260, ACM Press, 2005.

33. B. Mourrain, J.B. Lasserre, M. Laurent, P. Rostalski and P. Trebuchet, Moment matrices and border bases, In preparation.

34. P.A. Parrilo, Structured Semidefinite Programs and Semialgebraic Geometry Methods in Robustness and Optimization, Ph.D. thesis, California Institute of Technology, 2000.

35. G. Reid and L. Zhi, Solving polynomial systems via symbolic-numeric reduction to geometric involutive form, Journal of Symbolic Computation 44 (2009), no. 3, $280-291$.

36. P. Rostalski, Algebraic Moments - Real Root Finding and Related Topics, Ph.D. thesis, ETH Zürich, 2009.

37. , Bermeja: Convex Algebraic Geometry in MATLAB, Available from http://math. berkeley.edu/ philipp/cagwiki.

38. F. Rouillier, Solving zero-dimensional systems through the rational univariate representation, Journal of Applicable Algebra in Engineering, Communication and Computing 9 (1999), no. 5, 433-461.

39. N.Z. Shor, An approach to obtaining global extremums in polynomial mathematical programming problems, Kibernetica 5 (1987), 102-106.

40. A.J. Sommese and C.W. Wampler, The Numerical Solution of Systems of Polynomials Arising in Engineering and Science, World Scientific Press, 2005.

41. F. Sottile, Numerical real algebraic geometry, In preparation, 2009.

42. G. Stengle, A Nullstellensatz and a Positivstellensatz in semialgebraic geometry, Mathematische Annalen 207 (1974), 87-97. 
43. H.J. Stetter, Numerical Polynomial Algebra, SIAM, 2004.

44. B. Sturmfels, Solving Systems of Polynomial Equations, American Mathematical Society, 2002.

45. H. Wolkowicz, R. Saigal, and L. Vandenberghe (eds.), Handbook of Semidefinite Programming: Theory, Algorithms, and Applications, vol. 27, Kluwer Academic Publishers, Dordrecht, The Netherlands / Boston, MA, 2000. 